\title{
Spectral and Energy Efficiency of Superimposed Pilots in Uplink Massive MIMO
}

Daniel Verenzuela, Emil Björnson and Luca Sanguinetti

The self-archived postprint version of this journal article is available at Linköping University Institutional Repository (DiVA):

http://urn.kb.se/resolve?urn=urn:nbn:se:liu:diva-153185

N.B.: When citing this work, cite the original publication.

Verenzuela, D., Björnson, E., Sanguinetti, L., (2018), Spectral and Energy Efficiency of Superimposed Pilots in Uplink Massive MIMO, IEEE Transactions on Wireless Communications, 17(11), 7099-7115. https://doi.org/10.1109/TWC.2018.2860939

Original publication available at:

https://doi.org/10.1109/TWC.2018.2860939

Copyright: Institute of Electrical and Electronics Engineers (IEEE)

http://www.ieee.org/index.html

(C)2018 IEEE. Personal use of this material is permitted. However, permission to reprint/republish this material for advertising or promotional purposes or for creating new collective works for resale or redistribution to servers or lists, or to reuse any copyrighted component of this work in other works must be obtained from the IEEE. 


\title{
Spectral and Energy Efficiency of Superimposed Pilots in Uplink Massive MIMO
}

\author{
Daniel Verenzuela, Student Member, IEEE, Emil Björnson, Senior Member, IEEE, \\ Luca Sanguinetti, Senior Member, IEEE.
}

\begin{abstract}
Next generation wireless networks aim at providing substantial improvements in spectral efficiency (SE) and energy efficiency (EE). Massive MIMO has been proved to be a viable technology to achieve these goals by spatially multiplexing several users using many base station (BS) antennas. A potential limitation of Massive MIMO in multicell systems is pilot contamination, which arises in the channel estimation process from the interference caused by reusing pilots in neighboring cells. A standard method to reduce pilot contamination, known as regular pilot (RP), is to adjust the length of pilot sequences while transmitting data and pilot symbols disjointly. An alternative method, called superimposed pilot (SP), sends a superposition of pilot and data symbols. This allows to use longer pilots which, in turn, reduces pilot contamination. We consider the uplink of a multicell Massive MIMO network, with i.i.d. Rayleigh fading channels, using maximum ratio combining and compare RP and SP in terms of SE and EE. To this end, we derive rigorous closedform achievable rates with SP under a practical random BS deployment. We prove that the reduction of pilot contamination with SP is outweighed by the additional coherent and noncoherent interference. Numerical results show that when both methods are optimized, RP achieves comparable SE and EE to $S P$ in practical scenarios.
\end{abstract}

\section{INTRODUCTION}

The development of cellular networks is lead by the continuous increase in mobile data traffic [2]. The design of future cellular networks aims at handling $1000 \times$ more data traffic per unit area [3]. Meanwhile, the energy consumption of mobile communication systems is of great economical and ecological concerns [4]. Massive multiple-input multiple-output (MIMO) is considered as one of the most promising technologies to jointly improve spectral efficiency (SE) and energy efficiency (EE) [5]-[9]. The key idea of Massive MIMO is to utilize a large number of antennas (e.g., hundreds or thousands) at the base stations (BSs) to communicate coherently with several (e.g., tens or hundreds) user equipments (UEs) by virtue of spatial multiplexing [10]-[12].

The acquisition of channel state information (CSI) at the BS is essential in Massive MIMO. A time division duplexing

D. Verenzuela and E. Björnson are with the Department of Electrical Engineering (ISY), Linköping University, Linköping, SE-58183 Sweden (e-mail: daniel.verenzuela@liu.se; emil.bjornson@liu.se). L. Sanguinetti is with the Dipartimento di Ingegneria dell'Informazione, University of Pisa, Pisa, Italy, and with the Large Networks and System Group (LANEAS), CentraleSupélec, Université Paris-Saclay, Gif-sur-Yvette, France (e-mail: luca.sanguinetti@unipi.it).

This paper has received funding from ELLIIT and the Swedish Foundation for Strategic Research (SSF). The work of L. Sanguinetti was supported in part by ERC Starting MORE under Grant 305123.

A preliminary version [1] of this work was presented at IEEE GLOBECOM 2017.
(TDD) system is usually proposed to avoid the large overhead of downlink channel training and feedback [11], [12]. Uplink pilot sequences are transmitted by the UEs and channel reciprocity is exploited at the BS to coherently detect data from UEs in the uplink and precode data in the downlink. The time and frequency interval, over which the channel can be considered to remain static and frequency flat, called the coherence block, has a limited size and, in turn, there is a finite number of orthogonal pilot sequences that are available for channel estimation. Therefore, in multicell systems the pilot sequences need to be reused across cells. This creates coherent interference, called pilot contamination, between UEs that share the same pilots, which reduces the quality of channel estimates and affects the SE. The pilot contamination has been widely investigated in the literature. In [13]-[18], the same set of pilot sequences is assumed to be reused in all the cells and pilot contamination is mitigated by exploiting spatial channel correlation [12]-[15] or data covariance matrices [16]-[18]. In particular, [15] shows that the capacity grows without bound with the number of BS antennas when the covariance matrices of users sharing pilots are asymptotically linearly independent. In our work, uncorrelated Rayleigh fading is considered for reasons explained in Section II. Another approach is to have longer pilot sequences than the number of served UEs per cell to reduce the number of cells utilizing the same pilot [9], [19][21]. This method can effectively reduce pilot contamination at the cost of an increased estimation overhead that, in turn, decreases the amount of data symbols transmitted per coherence block. ${ }^{1}$ This tradeoff is studied in [20] under a hexagonal cell deployment and it turned out that a fraction between 5\% and $40 \%$ of the coherence block should be used for pilots.

In all the aforementioned works, the transmission of pilot and data symbols is done separately within the coherence block to reduce interference in the channel estimation process. This method is known in the literature as regular pilot (RP) transmission. In [22]-[28], the authors explore an alternative method that relies on the simultaneous transmission of pilot and data signals. This method is referred to as superimposed pilot (SP) and allows to increase the amount of samples that can be used for channel estimation and data transmission. By using SP, [22] proposes an optimal coherent receiver based on the Viterbi algorithm. Linear channel estimation methods of finite impulse response channels for single-input single-output

\footnotetext{
${ }^{1}$ This is a standard method in Massive MIMO literature and it is selected as the benchmark. Note that there are other methods to mitigate pilot contamination, however they often require high computational complexity or rely on specific channel properties.
} 
(SISO) systems are considered in [23] with only knowledge of the first order statistics. In [24], the authors compare SP and RP in a SISO system with Gauss-Markov flat fading channels, considering a practical setup, where channels change rapidly and UEs have low signal-to-noise ratios (SNRs). The results show that SP provides better performance than RP in terms of uncoded bit-error-rate and mean squared error (MSE) of channel estimates. Similar results have been found for stationary MIMO fading channels in [25]. In the aforementioned works [22]-[25], the authors focus on a single cell or single user scenario. Recently, [26]-[28] have shown that SP achieves promising results in multicell Massive MIMO systems. In particular, UEs transmit a linear combination of pilot and data symbols within the whole coherence block. This allows the use of longer pilot sequences, which can thus be reused less frequently in the network. Hence, pilot contamination is reduced, which could, in principle, improve the SE. However, sending pilot and data signals simultaneously causes interference in the channel estimation process from data symbols. This degrades the estimation quality and creates correlation between channel estimates and data. Moreover, the use of longer pilots increases the computational complexity of channel estimation and data detection. This, in turn, consumes more power and may eventually reduce the EE of the network. In summary, the use of SP in Massive MIMO systems introduces new sources of interference and increases the consumed power. All this may limit the practical gains of SP methods in terms of SE and $\mathrm{EE}$.

The aim of this paper is to evaluate the performance of SP in the uplink of a multicell Massive MIMO system and make comparisons with RP. To this end, we derive rigorous closed-form rate expressions with SP when using maximum ratio combining (MRC). This stands in contrast to prior works, [26], [28], which deal with approximate expressions of signalto-interference-plus-noise ratios (SINRs) and MSE of channel estimates. The analysis provided in this paper is valid for any number of BS antennas (not just for a large number). These formulas provide valuable insights into identifying all the interference sources, their impact on the SE and their relationship with the other system parameters. Furthermore, these expressions are used to perform the asymptotic analysis (corresponding to the large number of BS antennas regime) of the network, which allows to identify the conditions under which either RP or SP provides higher rates. Then, in order to properly study the effect associated with intercell interference in a large practical network with an irregular BS deployment, we adopt the stochastic geometry framework developed in [8] wherein BSs are spatially distributed according to a homogeneous Poisson point process (PPP). Within this setting, we calculate closed-form lower bounds of the achievable rates averaged over the UEs' spatial distribution. This provides powerful insights into the interplay of system parameters without requiring the use of heavy numerical simulations. Such lower bounds are then used to compute the EE of the network with both RP and SP taking into account the power consumed by transmission and circuitry. Numerical results show that, when both methods are optimized, RP provides comparable $\mathrm{SE}$ and $\mathrm{EE}$ to SP in practical scenarios.
The remainder of this paper is organized as follows. Section II introduces the network model. In Section III, the channel estimation process with RP and SP is detailed whereas the achievable rates with MRC are computed in Section IV. Section V presents detailed analytical comparisons between RP and SP. In Section VI, the average achievable rates are first computed for a random network deployment (based on stochastic geometry) and then used for computing the EE. Section VII illustrates numerical results while Section VIII concludes our work.

Notation: We denote vectors by lower-case bold-face letters (e.g., $\mathbf{x}$ ) and matrices by bold-face capital letters (e.g., $\mathbf{X}$ ). ${ }^{2}$ The operators $\mathbb{E}\{\cdot\}$ and $\mathbb{E}\{\cdot \mid y\}$ represent expected value and expected value conditioned on a realization of the random variable $y,{ }^{3}$ respectively. The notation $|\cdot|$ represents the absolute value and $\|\cdot\|$ denotes the Euclidean norm. We denote the transpose, conjugate transpose and conjugate operators as $(\cdot)^{T},(\cdot)^{H}$ and $(\cdot)^{*}$, respectively. We denote by $\mathbf{I}_{M}$ the identity matrix of size $M \times M$ and $C \mathcal{N}(\cdot, \cdot)$ indicates a circularly symmetric complex Gaussian distribution. To denote the set of real and complex numbers we use $\mathbb{R}$ and $\mathbb{C}$, respectively, while $\mathfrak{R}(\cdot)$ is the real part. $\Gamma(\cdot)$ denotes the Gamma function.

\section{NeTwork MOdeL}

We consider the uplink of a multicell Massive MIMO network where each BS has $M$ antennas and serves $K$ singleantenna UEs. We define $\Phi_{D}$ the set containing all BSs, where $D$ denotes the density of BSs per unit area (measured in $\mathrm{BS} / \mathrm{km}^{2}$ ). Note that this definition does not require the BSs to be distributed in any specific manner. However, a stochastic geometry framework will be used later on in Section VI to model the BS distribution. Without loss of generality, the following analysis is focused on an arbitrary BS, denoted as $\mathrm{BS}_{0}$ serving UEs in cell 0 , and an arbitrary UE $k$ in cell 0 , denoted as $\mathrm{UE}_{0 k}$. We define $\Psi_{D}=\Phi_{D} \backslash\{0\}$ as the set containing all other BSs than $\mathrm{BS}_{0}$.

We consider a network with bandwidth $B_{\mathrm{W}}$. The communication channels are modeled as block fading where each channel is considered to be constant over a coherence block of time duration $T_{c}$ and bandwidth $B_{c}{ }^{4}$ The total bandwidth is equally divided among all coherence blocks, which means that $B_{\mathrm{W}} / B_{c}$ is an integer number, and each block contains $\tau_{c}=B_{c} T_{c}$ complex samples. We assume uncorrelated Rayleigh fading channels since this is the first rigorous capacity analysis with $\mathrm{SP}$ in a multicell scenario. As done with RP, we believe that it is helpful to first develop fundamental theory for uncorrelated channels and then to extend it to correlated channels. Therefore, this is left for future work. Moreover, since uncorrelated fading corresponds to the worst-case scenario for pilot contamination and SP aims at mitigating this effect, this analysis gives insights into the main benefits of SP. In addition, the achievable rates under uncorrelated Rayleigh fading are

\footnotetext{
${ }^{2}[\mathbf{x}]_{j}$ is the $j^{\text {th }}$ element of $\mathbf{x}$. $[\mathbf{X}]_{j}$ denotes the $j^{\text {th }}$ column of $\mathbf{X}$, and $[\mathbf{X}]_{i j}$ is the $i^{\text {th }}$ row and $j^{\text {th }}$ column element of $\mathbf{X}$.

${ }^{3}$ We abuse the notation in conditional expectations by referring to the random variable and its realization with the same letter.

${ }^{4}$ In an OFDM system, the coherence bandwidth $B_{c}$ includes several subcarriers-see [11], [12] for more details.
} 


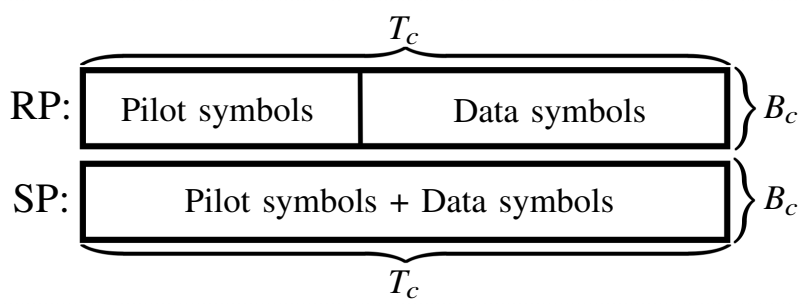

Fig. 1: Transmission protocol with RP and SP methods.

close to those under practical measured channels with nonline-of-sight and spatially distributed UEs [29]. We denote by $\mathbf{h}_{l l^{\prime} i} \in \mathbb{C}^{M}$ the channel between the $M$ antennas of $\mathrm{BS}_{l}$ and $\mathrm{UE}_{l^{\prime} i}$ in which the small-scale fading (SSF) is modeled as $\mathbf{h}_{l l^{\prime} i} \sim \mathcal{C N}\left(\mathbf{0}, \beta_{l l^{\prime} i} \mathbf{I}_{M}\right) \forall l, l^{\prime} \in \Phi_{D}$ and $i \in\{1, \ldots, K\}$ with $\beta_{l l^{\prime} i} \geq 0$ being the large-scale fading (LSF) coefficient between $\mathrm{BS}_{l}$ and $\mathrm{UE}_{l^{\prime} i}$. We assume that the distance between UEs and BSs is large enough to consider $\beta_{l l^{\prime} i}$ to be the same for all BS antennas. The received signal $\mathbf{y}_{0} \in \mathbb{C}^{M}$ at $\mathrm{BS}_{0}$ is

$$
\mathbf{y}_{0}=\sum_{l^{\prime} \in \Phi_{D}} \sum_{i=1}^{K} \mathbf{h}_{0 l^{\prime} i} \mathbf{x}_{l^{\prime} i}+\mathbf{n}_{0}
$$

where $\mathbf{n}_{0} \in \mathbb{C}^{M}$ is the noise vector distributed as $\mathbf{n}_{0} \sim \mathcal{C N}\left(\mathbf{0}, \sigma^{2} \mathbf{I}_{M}\right)$ and $\mathrm{x}_{l^{\prime} i}$ represents the transmitted signal from $\mathrm{UE}_{l^{\prime} i}$ in one arbitrary sample of the coherence block. The transmitted signal can be used for data, pilots or a superposition of the two depending on the employed method. We analyze the two transmission methods illustrated in Fig. 1: RP, called time-multiplexed in [26], and SP. With RP, data and pilot symbols are transmitted separately in each coherence block. Therefore, $\mathrm{x}_{l^{\prime} i}$ contains only one of the two in each sample of the coherence block. With SP, pilot and data symbols are transmitted simultaneously during the whole coherence block and thus $\mathrm{x}_{l^{\prime} i}$ contains a superposition of the two in each sample. ${ }^{5}$

\section{Channel Estimation}

To estimate the channels, we use standard linear minimum mean squared error (LMMSE) techniques [31] with both RP and SP.

\section{A. Regular pilots}

We consider a transmission protocol where $\tau_{p}$ out of the $\tau_{c}$ samples in each coherence block are reserved for pilot sequences, which leaves a fraction $1-\tau_{p} / \tau_{c}$ of samples for data transmission. We consider a set of $\tau_{p}$ orthogonal pilot sequences of length $\tau_{p}$. Each BS allocates $K \leq \tau_{p}$ different pilot sequences to the UEs served in its cell. We denote by $\phi_{l^{\prime} i} \in \mathbb{C}^{\tau_{p}}, \forall l^{\prime} \in \Phi_{D}, i \in\{1, \ldots, K\}$ the pilot sequence assigned to $\mathrm{UE}_{l^{\prime} i}$ with $\left|\left[\boldsymbol{\phi}_{l^{\prime} i}\right]_{j}\right|=1, \forall j \in\left\{1, \ldots, \tau_{p}\right\}$. To identify the UEs in different cells that share the same pilot as $\mathrm{UE}_{0 k}$ (including $\mathrm{UE}_{0 k}$ ), we define the set $\mathcal{P}_{0 k}^{\mathrm{RP}}=\left\{\left\{l^{\prime}, i\right\}: \boldsymbol{\phi}_{0 k}^{H} \boldsymbol{\phi}_{l^{\prime} i} \neq 0\right\}$.

\footnotetext{
${ }^{5}$ Note that superimposing more than one data symbol can increase the data rates by means of successive decoding algorithms [30]. However, in Massive MIMO the gains are marginal compared to the spatial multiplexing and array gains. Thus, this approach is not considered in the article.
}

$\mathrm{UE}_{0 k}$ transmits its pilot sequence $\boldsymbol{\phi}_{0 k}^{T}$ along with all other UEs in the network over $\tau_{p}$ instances of (1). At $\mathrm{BS}_{0}$, this yields the received signal $\mathbf{Z}_{0}^{\mathrm{RP}} \in \mathbb{C}^{M \times \tau_{p}}$ given by

$$
\mathbf{Z}_{0}^{\mathrm{RP}}=\sum_{l^{\prime} \in \Phi_{D}} \sum_{i=1}^{K} \sqrt{q_{l^{\prime} i}} \mathbf{h}_{0 l^{\prime} i} \boldsymbol{\phi}_{l^{\prime} i}^{T}+\overline{\mathbf{N}}_{0}
$$

where $q_{l^{\prime} i}$ is the transmission power of the pilot symbols from $\mathrm{UE}_{l^{\prime} i}$ and $\overline{\mathbf{N}}_{0}$ is the noise matrix with i.i.d. elements distributed as $\left[\overline{\mathbf{N}}_{0}\right]_{m j} \sim \mathcal{C N}\left(0, \sigma^{2}\right) \forall m \in\{1, \ldots, M\}, j \in\left\{1, \ldots, \tau_{p}\right\}$ with $\sigma^{2}$ being the noise variance. By multiplying $\mathbf{Z}_{0}^{\mathrm{RP}}$ with $\boldsymbol{\phi}_{0 k}^{*} / \sqrt{\tau_{p}}$, the received pilot signal is correlated with the pilot sequence corresponding to $\mathrm{UE}_{0 k}$, which is equivalent to despreading the received signal. This operation yields $\mathbf{z}_{0 k}^{\mathrm{RP}} \in \mathbb{C}^{M}$ given by

$$
\mathbf{z}_{0 k}^{\mathrm{RP}}=\mathbf{Z}_{0}^{\mathrm{RP}} \frac{\boldsymbol{\phi}_{0 k}^{*}}{\sqrt{\tau_{p}}}=\sum_{\left\{l^{\prime}, i\right\} \in \mathcal{P}_{0 k}^{\mathrm{RP}}} \sqrt{q l^{\prime} i \tau_{p}} \mathbf{h}_{0 l^{\prime} i}+\overline{\mathbf{n}}_{0}
$$

where $\overline{\mathbf{n}}_{0}=\overline{\mathbf{N}}_{0} \boldsymbol{\phi}_{0 k}^{*} / \sqrt{\tau_{p}}$ is a noise vector distributed as $\overline{\mathbf{n}}_{0} \sim C \mathcal{N}\left(\mathbf{0}, \sigma^{2} \mathbf{I}_{M}\right)$. Notice that no useful information is lost in the despreading operation, given that any signal in the orthogonal complement of $\boldsymbol{\phi}_{0 k}$ is independent of $\mathbf{z}_{0 k}^{\mathrm{RP}}$. Therefore, $\mathbf{z}_{0 k}^{\mathrm{RP}}$ in (3) is a sufficient statistic for estimating the channel $\mathbf{h}_{00 k}$ between $\mathrm{BS}_{0}$ and $\mathrm{UE}_{0 k}$. The minimum mean squared error (MMSE) estimate of $\mathbf{h}_{00 k}$ is given by the next lemma.

Lemma 1. With RP, the MMSE estimate of $\mathbf{h}_{00 k}$ is

$$
\hat{\mathbf{h}}_{00 k}=\frac{\bar{\gamma}_{0 k}^{\mathrm{RP}}}{\sqrt{q_{0 k} \tau_{p}}} \mathbf{z}_{0 k}^{\mathrm{RP}}
$$

with

$$
\bar{\gamma}_{0 k}^{\mathrm{RP}}=\frac{q_{0 k} \tau_{p} \beta_{00 k}}{\sum_{\left\{l^{\prime}, i\right\} \in \mathcal{P}_{0 k}^{\mathrm{RP}}} q_{l^{\prime} i} \tau_{p} \beta_{0 l^{\prime} i}+\sigma^{2}}
$$

and has covariance matrix given by

$$
\mathbb{E}\left\{\hat{\mathbf{h}}_{00 k} \hat{\mathbf{h}}_{00 k}^{H}\right\}=\beta_{00 k} \bar{\gamma}_{0 k}^{\mathrm{RP}} \mathbf{I}_{M} .
$$

Proof: It follows from applying standard LMMSE techniques [31, Ch. 12] to the problem at hand. Since $\mathbf{z}_{0 k}^{\mathrm{RP}}$ contains a Gaussian unknown signal plus independent Gaussian interference and noise, the LMMSE estimator coincides with the true MMSE estimator.

The parameter $\bar{\gamma}_{0 k}^{\mathrm{RP}} \in[0,1]$ indicates the quality of channel estimates. Notice that, as the length $\tau_{p}$ of the pilot sequences increases, $\bar{\gamma}_{0 k}^{\mathrm{RP}}$ also increases since the noise term becomes less significant and the cardinality of $\mathcal{P}_{0 k}^{\mathrm{RP}}$ decreases with $\tau_{p}$. This means that, as $\tau_{p}$ increases, the variance of the channel estimates approaches the variance of the true channels and estimation errors vanish. However, in practical applications $\tau_{p} \leq \tau_{c}$. Since $\tau_{c}$ is limited by the physical properties of the channel, there will always be an estimation error due to pilot contamination and noise. The key point to notice is that for scenarios where $\tau_{c}$ is much larger than $K$, the channel estimates with RP can be improved by letting $\tau_{p}$ be larger than $K$. 


\section{B. Superimposed pilots}

With SP, all the $\tau_{c}$ samples of the coherence block are used for transmitting pilot and data symbols. We consider $\tau_{c}$ orthogonal pilot sequences of length $\tau_{c}$ samples. Each BS selects $K \leq \tau_{c}$ different pilots and assigns them to its UEs. We denote by $\varphi_{l^{\prime} i} \in \mathbb{C}^{\tau_{c}}, \forall l^{\prime} \in \Phi_{D}, i \in\{1, \ldots, K\}$ the pilot sequence assigned to $\mathrm{UE}_{l^{\prime} i}$ with $\left|\left[\varphi_{l^{\prime} i}\right]_{j}\right|=1, \forall j \in\left\{1, \ldots, \tau_{c}\right\}{ }^{6}$ The set $\mathcal{P}_{0 k}^{\mathrm{SP}}=\left\{\left\{l^{\prime}, i\right\}: \boldsymbol{\varphi}_{0 k}^{H} \boldsymbol{\varphi}_{l^{\prime} i} \neq 0\right\}$ contains the indices of the UEs using the same pilot as $\mathrm{UE}_{0 k}$ (including $\mathrm{UE}_{0 k}$ ). $\mathrm{UE}_{0 k}$ transmits a superposition of the pilot sequence $\varphi_{0 k}^{T}$ and the data signal $\mathbf{s}_{0 k}^{T}$ along with all other UEs in the network over $\tau_{c}$ instances of (1). This yields an $M \times \tau_{c}$ received signal at $\mathrm{BS}_{0}$ given by

$$
\mathbf{Z}_{0}^{\mathrm{SP}}=\sum_{l^{\prime} \in \Phi_{D}} \sum_{i=1}^{K} \sqrt{q_{l^{\prime} i}} \mathbf{h}_{0 l^{\prime} i} \varphi_{l^{\prime} i}^{T}+\sum_{l^{\prime} \in \Phi_{D}} \sum_{i=1}^{K} \sqrt{p_{l^{\prime} i}} \mathbf{h}_{0 l^{\prime} i} \mathbf{s}_{l^{\prime} i}^{T}+\mathbf{N}_{0}
$$

where $p_{l^{\prime} i}$ and $q_{l^{\prime} i}$ are the transmission powers of the data and pilot symbols, respectively, transmitted by $\mathrm{UE}_{l^{\prime} i}$. The vector $\mathbf{s}_{l^{\prime} i} \in \mathbb{C}^{\tau_{c}}$ contains the data symbols transmitted in the whole coherence block. We assume the data symbols to be i.i.d. as $\mathbf{s}_{l^{\prime} i} \sim \mathcal{C N}\left(\mathbf{0}, \mathbf{I}_{\tau_{c}}\right)$. The noise matrix is defined as $\mathbf{N}_{0}=\left[\mathbf{n}_{01}, \ldots, \mathbf{n}_{0 \tau_{c}}\right]$ with i.i.d. columns distributed as $\mathbf{n}_{0 j} \sim \mathcal{C N}\left(0, \sigma^{2} \mathbf{I}_{M}\right) \forall j \in\left\{1, \ldots, \tau_{c}\right\}$. By multiplying $\mathbf{Z}_{0}^{\mathrm{SP}}$ with $\varphi_{0 k}^{*} / \sqrt{\tau_{c}}$, we obtain

$$
\begin{aligned}
\mathbf{z}_{0 k}^{\mathrm{SP}} & =\mathbf{Z}_{0}^{\mathrm{SP}} \frac{\boldsymbol{\varphi}_{0 k}^{*}}{\sqrt{\tau_{c}}}=\sum_{\left\{l^{\prime}, i\right\} \in \mathcal{P}_{0 k}^{\mathrm{SP}}} \sqrt{q_{l^{\prime} i} \tau_{c}} \mathbf{h}_{0 l^{\prime} i} \\
& +\sum_{l^{\prime} \in \Phi_{D}} \sum_{i=1}^{K} \sqrt{\frac{p_{l^{\prime} i}}{\tau_{c}}} \mathbf{h}_{0 l^{\prime} i} \mathbf{s}_{l^{\prime} i}^{T} \boldsymbol{\varphi}_{0 k}^{*}+\sum_{j=1}^{\tau_{c}} \mathbf{n}_{0 j} \frac{\left[\boldsymbol{\varphi}_{0 k}\right]_{j}^{*}}{\sqrt{\tau_{c}}}
\end{aligned}
$$

which is then used to compute the LMMSE estimate of the channel between $\mathrm{BS}_{0}$ and $\mathrm{UE}_{0 k}$.

Lemma 2. With SP, the LMMSE estimate of the channel $\mathbf{h}_{00 k}$ is

$$
\hat{\mathbf{h}}_{00 k}=\frac{\bar{\gamma}_{0 k}^{\mathrm{SP}}}{\sqrt{q_{0 k} \tau_{c}}} \mathbf{z}_{0 k}
$$

where

$$
\bar{\gamma}_{0 k}^{\mathrm{SP}}=\frac{q_{0 k} \tau_{c} \beta_{00 k}}{\sum_{\left\{l^{\prime}, i\right\} \in \mathcal{P}_{0 k}^{\mathrm{SP}}} q_{l^{\prime} i} \tau_{c} \beta_{0 l^{\prime} i}+\sum_{l^{\prime} \in \Phi_{D}} \sum_{i=1}^{K} p_{l^{\prime} i} \beta_{0 l^{\prime} i}+\sigma^{2}} .
$$

The covariance matrix of $\hat{\mathbf{h}}_{00 k}$ is

$$
\mathbb{E}\left\{\hat{\mathbf{h}}_{00 k} \hat{\mathbf{h}}_{00 k}^{H}\right\}=\bar{\gamma}_{0 k}^{\mathrm{SP}} \beta_{00 k} \mathbf{I}_{M}
$$

Proof: It follows from applying standard LMMSE estimation techniques [31, Ch. 12] to the problem at hand.

The parameter $\bar{\gamma}_{0 k}^{\mathrm{SP}} \in[0,1]$ indicates the quality of the channel estimates. From (10), it follows that the interference caused by data symbols is $\tau_{c}$-times less influential than the pilot interference from UEs that use the same pilot as $\mathrm{UE}_{0 k}$.

\footnotetext{
${ }^{6}$ Note that since the modulus of each pilot symbol is one, the peak-toaverage power ratio of the transmitted SP signal does not increase when adding the pilot symbols.
}

Moreover, as the length $\tau_{c}$ of the pilot sequences increases, $\bar{\gamma}_{0 k}^{\mathrm{SP}}$ approaches one since the data interference and noise become less influential and the cardinality of $\mathcal{P}_{0 k}^{\mathrm{SP}}$ decreases with $\tau_{c}$. This means that the variance of the channel estimates approaches the variance of the true channels. However, in practical applications $\tau_{c}$ is limited and thus there will always be an estimation error due to pilot contamination as well as interference from data signals and noise.

Remark 1. The key difference between the channel estimates with $R P$ and SP, apart from the number of observations $\left(\tau_{p}\right.$ with $R P$ and $\tau_{c}$ with $S P$ ), is the presence of extra interference with $S P$ due to the received data symbols (see the second term in the right-hand-side of (8)). This interference not only reduces the quality of the channel estimates but it also:

- Changes the distribution of the channel estimates. The received signal $\mathbf{z}_{0 k}^{\mathrm{SP}}$ in (8) is not Gaussian. Thus, the LMMSE estimate does not coincide with the true MMSE estimate and the channel estimates are only uncorrelated to the channel estimation errors but not independent, which stands in contrast to RP.

- Creates correlation between channel estimates and received data symbols from all UEs.

These phenomena play a key role in the achievable rate analysis with SP and create extra interfering terms that cannot be obtained from the closed-form expressions provided in [11], [12].

\section{AChiEvable Rates With MRC}

To evaluate the performance of the network, we derive ergodic achievable rates by applying standard lower bounding techniques on the capacity (e.g., [11], [12]). Since we consider a fixed bandwidth, the SE is obtained simply by scaling the achievable rates with $1 / B_{\mathrm{W}}$. We assume that MRC is employed for data detection. Particularly, the estimates of the data symbols transmitted by $\mathrm{UE}_{0 k}$ are obtained at $\mathrm{BS}_{0}$ by the inner product $\mathbf{v}_{00 k}^{H} \mathbf{y}_{0}$ with $\mathbf{v}_{00 k}=v_{00 k} \hat{\mathbf{h}}_{00 k}$, where $v_{00 k}=\frac{1}{\bar{\gamma}_{0 k}^{\mathrm{RP}} \sqrt{M \beta_{00 k}}}$ with RP and $v_{00 k}=\frac{1}{\bar{y}_{0 k}^{\mathrm{SP}} \sqrt{M \beta_{00 k}}}$ with SP. These scaling factors are selected to provide an equivalent gain of $M \beta_{00 k}$ for the desired signal with both methods.

To motivate the use of MRC, note that as $M \rightarrow \infty$, the directions of the channels $\mathbf{h}_{l l^{\prime} i} /\left\|\mathbf{h}_{l l^{\prime} i}\right\|$ of different UEs become asymptotically orthogonal. This is known as asymptotically favorable propagation [11], [12]. The squared norm of the channel scaled by $1 / M$ converges to a deterministic number, which is known as channel hardening [11], [12]. When considering uncorrelated Rayleigh fading, these phenomena make the use of linear detection techniques like MRC asymptotically optimal as $M \rightarrow \infty$ [11]. In addition, MRC has low complexity and thereby low consumed power.

\section{A. Random Pilot allocation}

The key advantage that SP has with respect to RP is the ability to use the whole coherence block for both channel estimation and data detection. To obtain clear insights into the data rate performance with respect to the number of samples used for channel estimation, $\tau_{p}$ (with RP) and $\tau_{c}$ (with SP), 
we consider a random pilot allocation method with both RP and SP. In particular, we assume that each BS selects $K$, out of $\tau_{p}$ (with RP) or $\tau_{c}$ (with SP), distinct pilot sequences uniformly at random in each coherence block and allocates them to its served UEs. We define $\chi_{l^{\prime} i}^{\mathrm{RP}}=\frac{\boldsymbol{\phi}_{0 k}^{H} \boldsymbol{\phi}_{l^{\prime} i}}{\tau_{p}} \in\{0,1\}$ and $\chi_{l^{\prime} i}^{\mathrm{SP}}=\frac{\varphi_{0 k}^{H} \varphi_{l^{\prime} i}}{\tau_{c}} \in\{0,1\}$ as binary random variables to indicate if $\mathrm{UE}_{l^{\prime} i}$ has the same pilot as $\mathrm{UE}_{0 k}$ with RP and SP, respectively. Notice that BSs allocate pilots independently and that UEs within each cell have different pilots, so that only one UE in cell $l^{\prime} \neq 0$ can have the same pilot as $\mathrm{UE}_{0 k}$. This means that for $l^{\prime} \neq 0, \sum_{i=1}^{K} \chi_{l^{\prime} i}^{\mathrm{RP}}$ and $\sum_{i=1}^{K} \chi_{l^{\prime} i}^{\mathrm{SP}}$ are Bernoulli distributed random variables with success probability $K / \tau_{p}$ and $K / \tau_{c}$, respectively. Thus, the following results hold:

$$
\begin{aligned}
\mathbb{E}\left\{\sum_{\left\{l^{\prime}, i\right\} \in \mathcal{P}_{0 k}^{\mathrm{RP} \backslash\{0, k\}}} q_{l^{\prime} i} \beta_{0 l^{\prime} i}\right\} & =\mathbb{E}\left\{\sum_{l^{\prime} \in \Psi_{D}} \sum_{i=1}^{K} \chi_{l^{\prime} i}^{\mathrm{RP}} q_{l^{\prime} i} \beta_{0 l^{\prime} i}\right\} \\
& =\sum_{l^{\prime} \in \Psi_{D}} \frac{K}{\tau_{p}} \frac{1}{K}\left(\sum_{i=1}^{K} q_{l^{\prime} i} \beta_{0 l^{\prime} i}\right) \\
\mathbb{E}\left\{\sum_{\left.\left\{l^{\prime}, i\right\} \in \mathcal{P}_{0 k}^{\mathrm{SP} \backslash\{0, k\}} q_{l^{\prime} i} \beta_{0 l^{\prime} i}\right\}}\right. & =\mathbb{E}\left\{\sum_{l^{\prime} \in \Psi_{D}} \sum_{i=1}^{K} \chi_{l^{\prime} i}^{\mathrm{SP}} q_{l^{\prime} i} \beta_{0 l^{\prime} i}\right\} \\
& =\sum_{l^{\prime} \in \Psi_{D}} \frac{K}{\tau_{c}} \frac{1}{K}\left(\sum_{i=1}^{K} q_{l^{\prime} i} \beta_{0 l^{\prime} i}\right)
\end{aligned}
$$

which allow us to obtain achievable rate expressions that do not depend on the particular construction of the sets $\mathcal{P}_{0 k}^{\mathrm{RP}}$ and $\mathcal{P}_{0 k}^{\mathrm{SP}}$.

\section{B. Regular pilots}

The received signal at $\mathrm{BS}_{0}$ with $\mathrm{RP}$, for an arbitrary data symbol $j$ in the coherence block, is

$$
\mathbf{y}_{0 j}^{\mathrm{RP}}=\sum_{i=1}^{K} \sqrt{p_{0 i}} \mathbf{h}_{00 i}\left[\mathbf{s}_{0 i}\right]_{j}+\sum_{l^{\prime} \in \Psi_{D}} \sum_{i=1}^{K} \sqrt{p_{l^{\prime} i}} \mathbf{h}_{0 l^{\prime} i}\left[\mathbf{s}_{l^{\prime} i}\right]_{j}+\mathbf{n}_{0 j}
$$

where $\mathbf{n}_{0 j}$ is the noise vector distributed as $\mathbf{n}_{0 j} \sim \mathcal{C N}\left(\mathbf{0}, \sigma^{2} \mathbf{I}_{M}\right)$. To detect the data symbol from $\mathrm{UE}_{0 k}$, the received signal $\mathbf{y}_{0 j}^{\mathrm{RP}}$ is combined with $\mathbf{v}_{00 k}$ to obtain

$$
\begin{aligned}
& {\left[\hat{\mathbf{s}}_{0 k}\right]_{j}=\mathbf{v}_{00 k}^{H} \mathbf{y}_{0 j}^{\mathrm{RP}}=\sqrt{p_{0 k}} \mathbb{E}\left\{\mathbf{v}_{00 k}^{H} \mathbf{h}_{00 k}\right\}\left[\mathbf{s}_{0 k}\right]_{j}} \\
& +\sqrt{p_{0 k}}\left(\mathbf{v}_{00 k}^{H} \mathbf{h}_{00 k}-\mathbb{E}\left\{\mathbf{v}_{00 k}^{H} \mathbf{h}_{00 k}\right\}\right)\left[\mathbf{s}_{0 k}\right]_{j}+\sum_{i \neq k}^{K} \sqrt{p_{0 i}} \mathbf{v}_{00 k}^{H} \mathbf{h}_{00 i}\left[\mathbf{s}_{0 i}\right]_{j} \\
& +\sum_{l^{\prime} \in \Psi_{D}} \sum_{i=1}^{K} \sqrt{p_{l^{\prime} i}} \mathbf{v}_{00 k}^{H} \mathbf{h}_{0 l^{\prime} i}\left[\mathbf{s}_{l^{\prime} i}\right]_{j}+\mathbf{v}_{00 k}^{H} \mathbf{n}_{0 j} .
\end{aligned}
$$

By treating the term $\sqrt{p_{0 k}} \mathbb{E}\left\{\mathbf{v}_{00 k}^{H} \mathbf{h}_{00 k}\right\}\left[\mathbf{s}_{0 k}\right]_{j}$ as the desired signal and the remaining ones in (15) as effective noise, we have an equivalent SISO system with a deterministic channel and non-Gaussian effective noise, which is uncorrelated with the data symbol $\left[\mathbf{s}_{0 k}\right]_{j}$. Moreover, the individual terms in the effective noise (second to last terms in (15)) are also uncorrelated due to the fact that the data symbols from different UEs have zero mean and are independent among themselves and independent from the noise. In the next lemma, we provide an ergodic achievable rate, i.e., a lower bound on the capacity, of the system when using RP.

Lemma 3. An ergodic achievable rate for $\mathrm{UE}_{0 k}$ with $R P$ and $M R C$ is

$$
\mathrm{R}_{0 k}^{\mathrm{RP}}=B_{\mathrm{W}}\left(1-\frac{\tau_{p}}{\tau_{c}}\right) \log _{2}\left(1+\mathrm{SINR}_{0 k}^{\mathrm{RP}}\right)
$$

where $\mathrm{SINR}_{0 k}^{\mathrm{RP}}$ is the effective SINR of $\mathrm{UE}_{0 k}$ given by

$$
\begin{aligned}
& =\frac{\operatorname{SINR}_{0 k}^{\mathrm{RP}}}{\sum_{l^{\prime} \in \Phi_{D}} \sum_{i=1}^{K} p_{l^{\prime} i} \mathbb{E}\left\{\left|\mathbf{v}_{00 k}^{H} \mathbf{h}_{0 l^{\prime} i}\right|^{2}\right\}-\left|\mathbb{E}\left\{\mathbf{v}_{00 k}^{H} \mathbf{h}_{00 k}\right\}\right|^{2}+\mathbb{E}\left\{\left|\mathbf{v}_{00 k}^{H} \mathbf{n}_{0}\right|^{2}\right\}} \\
& =\frac{M p_{0 k} \beta_{00 k}}{\frac{M}{\tau_{p}} \sum_{l^{\prime} \in \Psi_{D}} \sum_{i=1}^{K} \frac{p_{l^{\prime} i} q_{l^{\prime} i}}{q_{0 k}} \frac{\beta_{0 l^{\prime} i}^{2}}{\beta_{00 k}}+\frac{1}{\gamma_{0 k}^{\mathrm{RP}}}\left(\sum_{l^{\prime} \in \Phi_{D}} \sum_{i=1}^{K} p_{l^{\prime} i} \beta_{0 l^{\prime} i}+\sigma^{2}\right)}
\end{aligned}
$$

and

$$
\gamma_{0 k}^{\mathrm{RP}}=\mathbb{E}\left\{\frac{1}{\bar{\gamma}_{0 k}^{\mathrm{RP}}}\right\}^{-1}=\frac{q_{0 k} \tau_{p} \beta_{00 k}}{q_{0 k} \tau_{p} \beta_{00 k}+\sum_{l^{\prime} \in \Psi_{D}} \sum_{i=1}^{K} q_{l^{\prime} i} \beta_{0 l^{\prime} i}+\sigma^{2}} .
$$

The expectations in (17) are taken with respect to the SSF and the random pilot allocation. Note that the ergodic achievable rate with effective SINR given by (17) holds for any selection of $\mathbf{v}_{00 k}$ and any channel distribution.

Proof: It follows from standard lower bounds [11, Ch. 2] on the capacity between the transmitter and receiver when only knowledge of the average effective channel $\mathbb{E}\left\{\mathbf{v}_{00 k}^{H} \mathbf{h}_{00 k}\right\}$ is used to obtain an equivalent SISO system with a deterministic channel and non-Gaussian effective noise. The closed-form expression of the SINR follows the same approach as in [8], [21], [11, Ch. 4] where the independence between the channel estimates and errors is used to compute the expectations in (17) in closed-form. In addition, the result in (12) is used to calculate the expectations with respect to $\chi_{l^{\prime} i}^{\mathrm{RP}}$.

To mitigate the effect of pilot contamination with RP, we can increase the pilot overhead by selecting $\tau_{p}>K$. This improves the quality of channel estimates (see Section III-A) and reduces the interference from pilot contamination (see first term in the denominator of (18)). This approach is simple and provides good results when a pilot reuse factor is used [11]. Thus, it provides a suitable comparison reference when evaluating the performance of SP. The selection of $\tau_{p}$ is of paramount importance in order to assess the performance of RP. Therefore, in Section VII we provide numerical results when $\tau_{p}$ is optimized to maximize the data rates. This optimization is done through an exhaustive search over the integer values of $\tau_{p} \in\left[K, \tau_{c}\right]$.

\section{Superimposed pilots}

In the case of SP, the received signal for an arbitrary data symbol $j$ in the coherence block, at $\mathrm{BS}_{0}$, is given by the $j^{\text {th }}$ 


$$
\begin{aligned}
& \operatorname{SINR}_{0 k}^{\mathrm{SP}}=\frac{p_{0 k}}{M \beta_{00 k}}\left|\mathbb{E}\left\{\left\|\mathbf{h}_{00 k}\right\|^{2}\right\}\right|^{2} /\left(\frac{p_{0 k}}{M \beta_{00 k}}\left(\mathbb{E}\left\{\left\|\mathbf{h}_{00 k}\right\|^{4}\right\}-\left|\mathbb{E}\left\{\left\|\mathbf{h}_{00 k}\right\|^{2}\right\}\right|^{2}\right)+\mathbb{E}\left\{\left|n_{\text {eff }}-\mathbb{E}\left\{n_{\text {eff }}\right\}\right|^{2}\right\}\right) \\
& =M p_{0 k} \beta_{00 k} /\left(\frac{M}{\tau_{c}} \sum_{l^{\prime} \in \Psi_{D}} \sum_{i=1}^{K} \frac{\left(p_{l^{\prime} i}+\left(1-\frac{1}{\tau_{c}}\right) q_{l^{\prime} i}\right) q_{l^{\prime} i}}{q_{0 k}} \frac{\beta_{0 l^{\prime} i}^{2}}{\beta_{00 k}}+\frac{M}{\tau_{c}} \sum_{l^{\prime} \in \Phi_{D}} \sum_{i=1}^{K} \frac{\left(p_{l^{\prime} i}+q_{l^{\prime} i}\right) p_{l^{\prime} i}}{q_{0 k}} \frac{\beta_{0 l^{\prime} i}^{2}}{\beta_{00 k}}\right. \\
& \text { Coherent interference }
\end{aligned}
$$

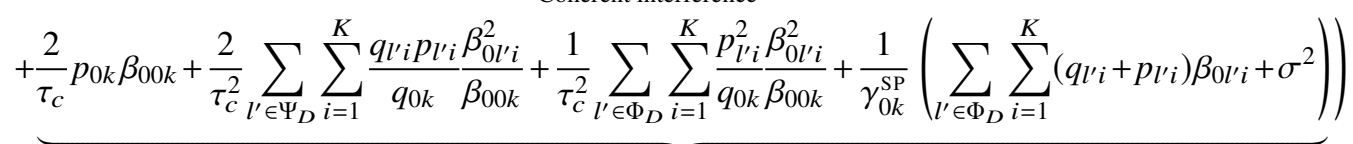

Non-coherent interference and noise

column of $\mathbf{Z}_{0}^{\mathrm{SP}}$ (see (7)). By combining the received signal $\left[\mathbf{Z}_{0}^{\mathrm{SP}}\right]_{j}$ with $\mathbf{v}_{00 k}$, an estimate of the data symbol $j$ transmitted by $\mathrm{UE}_{0 k}$ is obtained as $\left[\hat{\mathbf{s}}_{0 k}\right]_{j}=\mathbf{v}_{00 k}^{H}\left[\mathbf{Z}_{0}^{\text {SP }}\right]_{j}$. To compute an ergodic achievable rate, we first isolate the term that contains the desired information. To this end, we rewrite the combining vector as

$$
\mathbf{v}_{00 k}=v_{00 k} \bar{\gamma}_{0 k}^{\mathrm{SP}} \mathbf{h}_{00 k}+\overline{\mathbf{v}}_{00 k}=\frac{1}{\sqrt{M \beta_{00 k}}} \mathbf{h}_{00 k}+\overline{\mathbf{v}}_{00 k}
$$

where

$$
\begin{aligned}
\overline{\mathbf{v}}_{00 k} & =\frac{v_{00 k} \bar{\gamma}_{0 k}^{\mathrm{SP}}}{\sqrt{q_{0 k} \tau_{c}}}\left(\sum_{l^{\prime} \in \Psi_{D}} \sum_{i=1}^{K} \chi_{l^{\prime} i}^{\mathrm{SP}} \sqrt{q_{l^{\prime} i} \tau_{c}} \mathbf{h}_{0 l^{\prime} i}\right. \\
& \left.+\sum_{l^{\prime} \in \Phi_{D}} \sum_{i=1}^{K} \sqrt{\frac{p_{l^{\prime} i}}{\tau_{c}}} \mathbf{h}_{0 l^{\prime} i} \mathbf{s}_{l^{\prime} i}^{T} \boldsymbol{\varphi}_{0 k}^{*}+\sum_{j^{\prime}=1}^{\tau_{c}} \mathbf{n}_{0 j^{\prime}} \frac{\left[\boldsymbol{\varphi}_{0 k}\right]_{j^{\prime}}^{*}}{\sqrt{\tau_{c}}}\right) .
\end{aligned}
$$

Next, we add and subtract $\sqrt{\frac{p_{0 k}}{M \beta_{00 k}}} \mathbb{E}\left\{\left\|\mathbf{h}_{00 k}\right\|^{2}\right\}\left[\mathbf{s}_{0 k}\right]_{j}$ from the data estimate $\left[\hat{\mathbf{s}}_{0 k}\right]_{j}$ to obtain a desired signal with deterministic effective channel gain. This leads to

$$
\begin{aligned}
& {\left[\hat{\mathbf{s}}_{0 k}\right]_{j}=\sqrt{\frac{p_{0 k}}{M \beta_{00 k}}} \mathbb{E}\left\{\left\|\mathbf{h}_{00 k}\right\|^{2}\right\}\left[\mathbf{s}_{0 k}\right]_{j}} \\
& +\sqrt{\frac{p_{0 k}}{M \beta_{00 k}}}\left(\left\|\mathbf{h}_{00 k}\right\|^{2}-\mathbb{E}\left\{\left\|\mathbf{h}_{00 k}\right\|^{2}\right\}\right)\left[\mathbf{s}_{0 k}\right]_{j}+n_{e f f}
\end{aligned}
$$

where

$$
\begin{aligned}
& n_{e f f}=\sqrt{p_{0 k}} \overline{\mathbf{v}}_{00 k}^{H} \mathbf{h}_{00 k}\left[\mathbf{s}_{0 k}\right]_{j} \\
& +\sum_{l^{\prime} \in \Phi_{D}} \sum_{i=1}^{K}\left(\sqrt{q_{l^{\prime} i}}\left[\boldsymbol{\varphi}_{l^{\prime} i}\right]_{j}+\xi_{l^{\prime} i} \sqrt{p_{l^{\prime} i}}\left[\mathbf{s}_{l^{\prime} i}\right]_{j}\right) \mathbf{v}_{00 k}^{H} \mathbf{h}_{0 l^{\prime} i}+\mathbf{v}_{00 k}^{H} \mathbf{n}_{0 j} .
\end{aligned}
$$

The term $n_{\text {eff }}$ is defined in (23) for analytical tractability and accounts for the interference caused by pilot and data symbols received from all UEs (including self-interference from $\mathrm{UE}_{0 k}$ ) plus noise. For ease of notation, we define $\xi_{l^{\prime} i}=0$ for $\left\{l^{\prime}, i\right\}=\{0, k\}$ and $\xi_{l^{\prime} i}=1$ otherwise.

Notice that the first term in (22) is uncorrelated with the remaining ones in (22) since the data symbols have zero mean, are independent and circularly symmetric complex Gaussian. Thus, we have an equivalent SISO system with deterministic effective channel and non-Gaussian effective noise for which we can obtain an achievable rate based on the analysis in [11, Ch. 2]. This result is summarized in the following theorem.
Theorem 1. An ergodic achievable rate for $\mathrm{UE}_{0 k}$ with $S P$ and $M R C$ is

$$
\mathrm{R}_{0 k}^{\mathrm{SP}}=B_{\mathrm{W}} \log _{2}\left(1+\mathrm{SINR}_{0 k}^{\mathrm{SP}}\right)
$$

where $\mathrm{SINR}_{0 k}^{\mathrm{SP}}$ is the effective SINR of $\mathrm{UE}_{0 k}$ given in (26) and (27) at the top of the page with

$$
\begin{aligned}
& \gamma_{0 k}^{\mathrm{SP}}=\mathbb{E}\left\{\frac{1}{\bar{\gamma}_{0 k}^{\mathrm{SP}}}\right\}^{-1} \\
& =\frac{q_{0 k} \tau_{c} \beta_{00 k}}{q_{0 k} \tau_{c} \beta_{00 k}+\sum_{l^{\prime} \in \Psi_{D}} \sum_{i=1}^{K} q_{l^{\prime} i} \beta_{0 l^{\prime} i}+\sum_{l^{\prime} \in \Phi_{D}} \sum_{i=1}^{K} p_{l^{\prime} i} \beta_{0 l^{\prime} i}+\sigma^{2}} .
\end{aligned}
$$

The term $n_{\text {eff }}$ contains the last terms of the effective noise defined in (23). The expectations in (26) are taken with respect to the SSF and the random pilot allocation.

Proof: It follows from taking the estimate of $\left[\hat{\mathbf{s}}_{0 k}\right]_{j}$ in (22) and establishing an equivalent SISO system with a deterministic channel and uncorrelated non-Gaussian effective noise. Then, by applying standard lower bounds on the capacity between the transmitter and receiver of the equivalent SISO system, the ergodic achievable rate with effective SINR shown in (26) is derived [11, Ch. 2]. The proof for obtaining the closed-form expression in (27) can be found in Appendix A.

With SP, there is no pre-log factor in (24) since the whole coherence block is used for data transmission. The coherent gain (see the numerator of (27)) scales with $M$ and depends on the factor $\gamma_{0 k}^{\mathrm{SP}}$ (see (25)), which reflects the channel estimation quality. We define the coherent interference as the interference that adds constructively in the detection process due to the correlation between the combining vector and the received signal. As a result, its variance scales with $M$. With non-coherent interference, we refer to all the sources of interference that are combined non-constructively whose variance, in turn, does not scale with $M$. There is coherent interference from pilot contamination and also from pilot and data symbols (see the first two terms in the denominator of (27)) due to the correlation between channel estimates and data symbols. Similarly, there is non-coherent interference from pilot symbols, data symbols and cross-correlation of the two (see the third, fourth, and fifth terms in the denominator of (27)). In the prior works [26, Eq. (12)] and [28, Eq. (41)], approximate SINR expressions are provided with SP and 


$$
\operatorname{SINR}_{0 k}^{\mathrm{SP}-\mathrm{UB}}=\underbrace{\frac{M}{\tau_{c}} \sum_{l^{\prime} \in \Psi_{D}} \sum_{i=1}^{K} \frac{p_{l^{\prime} i} q_{l^{\prime} i}}{q_{0 k}} \frac{\beta_{0 l^{\prime} i}^{2}}{\beta_{00 k}}+\frac{M}{\tau_{c}} \sum_{l^{\prime} \in \Phi_{D}} \sum_{i=1}^{K} \frac{p_{l^{\prime} i}^{2}}{q_{0 k}} \frac{\beta_{0 l^{\prime} i}^{2}}{\beta_{00 k}}}_{\text {Coherent interference }}+\underbrace{\frac{1}{\tau_{c}^{2}} \sum_{l^{\prime} \in \Phi_{D}} \sum_{i=1}^{K} \frac{p_{l^{\prime} i}^{2}}{q_{0 k}} \frac{\beta_{0 l^{\prime} i}^{2}}{\beta_{00 k}}+\frac{1}{\gamma_{0 k}^{\mathrm{SP}}} \sum_{l^{\prime} \in \Phi_{D}} \sum_{i=1}^{\sum_{00 k}} \sum_{\left.p_{l^{\prime} i} \beta_{0 l^{\prime} i}+\sigma^{2}\right)}^{\sigma^{2}}}_{\text {Non-coherent interference and noise }}
$$

MRC based on asymptotic favorable propagation and channel hardening (i.e., $\lim _{M \rightarrow \infty} \frac{\mathbf{h}_{0 l i}^{H} \mathbf{h}_{0 l^{\prime} i^{\prime}}}{M}=0$ if $\{l, i\} \neq\left\{l^{\prime}, i^{\prime}\right\}$ and $\left.\lim _{M \rightarrow \infty} \frac{\left\|\mathbf{h}_{0 l i}\right\|^{2}}{M}=\beta_{0 l i}\right)$. In contrast, the result in Theorem 1 does not rely on any asymptotic approximation. This enables us to accurately analyze the system performance for any finite $M$. By comparing [26, Eq. (12)] and [28, Eq. (41)] with (27), it is seen that (27) contains extra interfering terms, which might greatly affect the system performance.

Notice that since the pilot symbols are known to the BSs, they can be subtracted from $n_{\text {eff }}$ to reduce the interference and obtain a better estimate of data symbols [26]. To obtain clear insights into the effect of the interference from pilot symbols, suppose the received pilot symbols can be perfectly removed from $n_{\text {eff. Let }}$

$$
\begin{aligned}
\bar{n}_{e f f}= & \sqrt{p_{0 k}} \overline{\mathbf{v}}_{00 k}^{H} \mathbf{h}_{00 k}\left[\mathbf{s}_{0 k}\right]_{j} \\
& +\sum_{l^{\prime} \in \Phi_{D}} \sum_{i=1}^{K} \xi_{l^{\prime} i} \sqrt{p_{l^{\prime} i}}\left[\mathbf{s}_{l^{\prime} i}\right]_{j} \mathbf{v}_{00 k}^{H} \mathbf{h}_{0 l^{\prime} i}+\mathbf{v}_{00 k}^{H} \mathbf{n}_{0 j}
\end{aligned}
$$

be the resulting term without the effect of pilot interference. Then, by replacing $n_{\text {eff }}$ with $\bar{n}_{\text {eff }}$ in (26) we can compute an upper bound on the effective SINR with SP. This is summarized in the following corollary.

Corollary 1. By removing the received pilot symbols perfectly from the data estimates, the effective SINR with SP is upper bounded as $\mathrm{SINR}_{0 k}^{\mathrm{SP}} \leq \mathrm{SINR}_{0 k}^{\mathrm{SP}-\mathrm{UB}}$ given in (28) at the top of the page.

Proof: It follows from replacing $n_{\text {eff }}$ with $\bar{n}_{\text {eff }}$ in (26) and deriving the closed-form expression with the same approach as in Appendix A.

By subtracting the received pilot symbols perfectly from $n_{\text {eff }}$, both the coherent and non-coherent interference are reduced and some of the cross terms in the non-coherent interference vanish. This can increase the data rates provided that the proportion of power used for pilot symbols is not negligible. However, in practice the pilot symbols cannot be perfectly removed from data estimates because channels are not perfectly known (see Section III-B). Alternatively, we can remove the estimates of the received pilot symbols (i.e., $\sum_{l^{\prime} \in \Phi_{D}} \sum_{i=1}^{K} \sqrt{q l^{\prime} i}\left[\varphi_{l^{\prime} i}\right]_{j} \mathbf{v}_{00 k}^{H} \hat{\mathbf{h}}_{0 l^{\prime} i}$ ) from $n_{\text {eff. }}$. This approach would introduce a large number of cross terms into the variance of $n_{\text {eff }}$ since the channel estimates are correlated with the received data symbols of all UEs (see Remark 1), and a closed-form expression of the effective SINR would not provide clear insights into the performance. The effect of removing the estimates of the received pilot symbols is evaluated numerically in Section VII.

Notice that iterative decoding algorithms can be used to improve channel and data estimates. This is achieved at the price of an increased computational complexity with SP since the number of operations in each iteration grows linearly with $M$ and $\tau_{c}$ [26]. Moreover, similar approaches can also be used with RP where the data estimates can be used to improve the channel estimates and vice versa. As the first capacity analysis with SP, we focus on MRC and use the results with perfect pilot subtraction (shown in Corollary 1) to evaluate the possible gains of more complex signal processing schemes. The use of iterative decoding algorithms is thus left for future work.

\section{ANAlysis OF ACHIEVABLE RATES}

To compare the rate expressions in Lemma 3, Theorem 1 and Corollary 1, we characterize the terms in the effective SINR expressions (18) with RP and (27), (28) with SP, and analyze their influence on the network performance. From Table I, we can see that by using the full coherence block for pilots in SP: $i$ ) the estimates improve when $\tau_{c}$ increases; ii) there is no penalty in the pre-log factor on the achievable rate; and iii) the pilot contamination is reduced by a factor of $1 / \tau_{c}$. However, due to the high correlation between the received signal $\left[\mathbf{Z}_{0}^{\mathrm{SP}}\right]_{j}$ and the channel estimate $\hat{\mathbf{h}}_{00 k}$, there are other interfering terms that are combined coherently or non-coherently. By subtracting perfectly the received pilot symbols, the coherent and non-coherent interference is reduced and several interference terms from cross products between pilot and data symbols vanish. The relative strengths of the interference terms depend on the network deployment setup.

To gain further insights, we consider the asymptotic limit when $M \rightarrow \infty$. This shows the influence of the interference that combines coherently in the detection process. The asymptotic limits are summarized in the following corollary.

Corollary 2. The achievable rates of $\mathrm{UE}_{0 k}$ with $R P$ and $S P$ when $M \rightarrow \infty$ are given by

$$
\mathrm{R}_{0 k}^{\mathrm{A}-\mathrm{RP}}=\left(1-\frac{\tau_{p}}{\tau_{c}}\right) B_{\mathrm{W}} \log _{2}\left(1+\frac{p_{0 k} \beta_{00 k}}{\frac{1}{\tau_{p}} \sum_{l^{\prime} \in \Psi_{D}} \sum_{i=1}^{K} \frac{p_{l^{\prime} i} q_{l^{\prime} i}}{q_{0 k}} \frac{\beta_{0 l^{\prime} i}^{2}}{\beta_{00 k}}}\right)
$$

and $\mathrm{R}_{0 k}^{\mathrm{A}-\mathrm{SP}}$ which is shown in (31), (32) in the page after next.

Proof: It follows from taking the limit in the expressions (18), (27) and (28).

The above asymptotic formulas can be used to compare RP and SP. We see that the scaling factor $1 / \tau_{p}$ in the coherent pilot contamination with RP (see the denominator of the fraction inside the logarithm in (30)) is larger than $1 / \tau_{c}$ with SP (see 


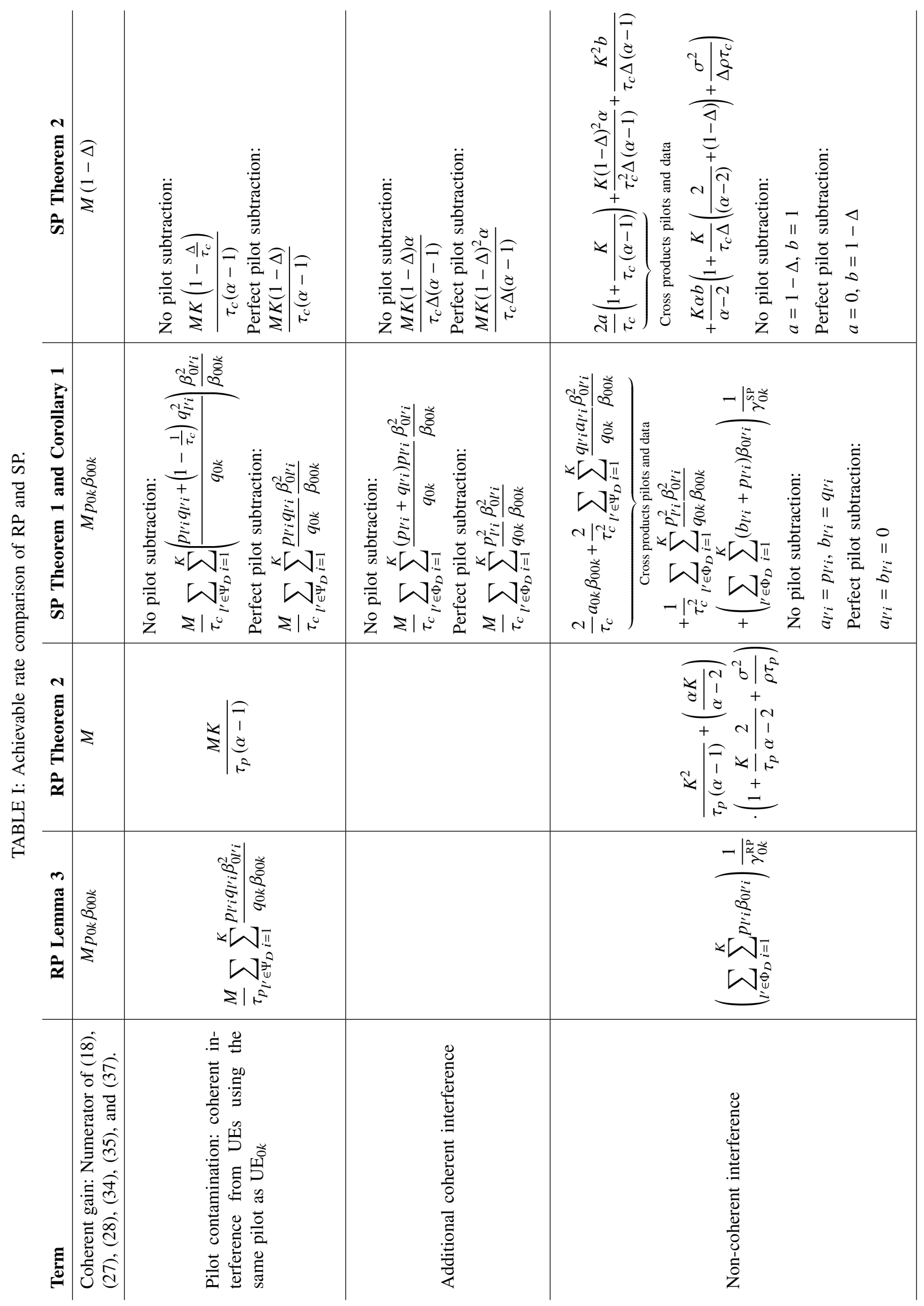




$$
\begin{aligned}
\mathrm{R}_{0 k}^{\mathrm{A}-\mathrm{SP}} & =B_{\mathrm{W}} \log _{2}\left(1+\frac{p_{0 k} \beta_{00 k}}{\frac{1}{\tau_{c}} \sum_{l^{\prime} \in \Psi_{D}} \sum_{i=1}^{K} \frac{\left(p_{l^{\prime} i}+\left(1-\frac{1}{\tau_{c}}\right) q_{l^{\prime} i}\right) q_{l^{\prime} i}}{q_{0 k}} \frac{\beta_{0 l^{\prime} i}^{2}}{\beta_{00 k}}+\frac{1}{\tau_{c}} \sum_{l^{\prime} \in \Phi_{D}} \sum_{i=1}^{K} \frac{\left(p_{l^{\prime} i}+q_{l^{\prime} i}\right) p_{l^{\prime} i}}{q_{0 k}} \frac{\beta_{0 l^{\prime} i}^{2}}{\beta_{00 k}}}\right) \\
& \leq B_{\mathrm{W}} \log _{2}\left(1+\frac{p_{0 k} \beta_{00 k}}{\frac{1}{\tau_{c}} \sum_{l^{\prime} \in \Psi_{D}} \sum_{i=1}^{K} \frac{p_{l^{\prime} i} q_{l^{\prime} i}}{q_{0 k}} \frac{\beta_{0 l^{\prime} i}^{2}}{\beta_{00 k}}+\frac{1}{\tau_{c}} \sum_{l^{\prime} \in \Phi_{D}} \sum_{i=1}^{K} \frac{p_{l^{\prime} i}^{2}}{q_{0 k}} \frac{\beta_{0 l^{\prime} i}^{2}}{\beta_{00 k}}}\right) .
\end{aligned}
$$

the denominator of the fraction inside the logarithm in (31), (32)). However, with SP there is another term with coherent interference that affects the performance. If we compare the results in Corollary 2 with [26, Eq. (13)] and [28, Eq. (42)], the following two differences are observed. First, the pilot contamination term with SP is neglected in [26], [28], which is a valid assumption only for scenarios wherein the total number of UEs in the entire network is lower than $\tau_{c}$. However, this is not the case of practical networks with many cells, and thus there will be pilot contamination also with SP. Secondly, in [26] the length of the pilot signals $\tau_{p}$ with RP is assumed not to change with the coherence block size. However, this parameter can indeed be optimized for a given size of the coherence block. As a result, with RP the effect of pilot contamination changes with the size of the coherence block as well and this could affect the scenarios in which SP outperforms RP, and vice versa.

To compare the asymptotic achievable rates given by (30) and (31), (32), we define $\zeta=\tau_{p} / \tau_{c}$ as the ratio between the pilot length with RP and the size of the coherence block. The value of $\zeta$ that maximizes the achievable rate with RP is given as follows.

Corollary 3. With RP, the asymptotic rate when $M \rightarrow \infty$ is a concave function of $\zeta$ and its maximum is found at

$$
\zeta^{\max }=\frac{1}{\operatorname{SIR}_{\mathrm{RP}}}\left(\frac{1+\operatorname{SIR}_{\mathrm{RP}}}{W\left(\left(1+\operatorname{SIR}_{\mathrm{RP}}\right) e\right)}-1\right) \in(0,1)
$$

for $\mathrm{SIR}_{\mathrm{RP}}>0$ where

$$
\operatorname{SIR}_{\mathrm{RP}}=\frac{p_{0 k} \beta_{00 k}}{\frac{1}{\tau_{c}} \sum_{l^{\prime} \in \Psi_{D}} \sum_{i=1}^{K} \frac{p_{l^{\prime} i} q_{l^{\prime} i}}{q_{0 k}} \frac{\beta_{0 l^{\prime} i}^{2}}{\beta_{00 k}}}
$$

and $W(\cdot)$ denotes the Lambert $W$ function ${ }^{7}$ and e denotes the base of the natural logarithm.

Proof: The corollary is proved in Appendix B.

Notice that: i) $\mathrm{R}_{0 k}^{\mathrm{A}-\mathrm{RP}}$ is a concave function of $\zeta \in[0,1]$ that starts $(\zeta=0)$ and ends $(\zeta=1)$ at zero and thus it is not monotonic; ii) $\mathrm{R}_{0 k}^{\mathrm{A}-\mathrm{RP}}$ depends linearly and logarithmically on $\zeta$. To the best of our knowledge, it is not possible to find in closed-form the solution to the inequality $\mathrm{R}_{0 k}^{\mathrm{A}-\mathrm{RP}} \leq \mathrm{R}_{0 k}^{\mathrm{A}-\mathrm{SP}}$ in terms of $\zeta$. Let $\mathrm{R}_{0 k}^{\mathrm{A}-\mathrm{RP}-\mathrm{MAX}}=\max _{\zeta \in[0,1]}\left\{\mathrm{R}_{0 k}^{\mathrm{A}-\mathrm{RP}}\right\}$, then

\footnotetext{
${ }^{7}$ The Lambert $W$ function is defined as $z=W(z) e^{W(z)}$ for any $z \in \mathbb{C}$. More details can be found in [32].
}

if $\mathrm{R}_{0 k}^{\mathrm{A}-\mathrm{SP}}>\mathrm{R}_{0 k}^{\mathrm{A}-\mathrm{RP}-\mathrm{MAX}}$ the asymptotic achievable rate with SP always outperforms RP. However, if $\mathrm{R}_{0 k}^{\mathrm{A}-\mathrm{SP}}<\mathrm{R}_{0 k}^{\mathrm{A}-\mathrm{RP}-\mathrm{MAX}}$ there exists an interval around $\zeta^{\max }$ for which the asymptotic achievable rate with RP is better than SP. Since the inequality condition $\mathrm{R}_{0 k}^{\mathrm{A}-\mathrm{SP}}>\mathrm{R}_{0 k}^{\mathrm{A}-\mathrm{RP}}$ depends on the power allocation and LSF coefficients, we need to consider a particular network deployment setup to offer a more precise comparison between RP and SP. This is what we do in the next sections.

\section{PERformance COMPARISON UNDER RANDOM DEPLOYMENT}

As shown in Sections IV and V, it is necessary to have a particular network deployment setup to obtain further insights into the performance of SP and RP. To model the irregularity and large number of cells of practical networks, we use the stochastic geometry framework from [8], which has been shown to accurately model real network deployments [33]. Here, the BSs are distributed according to a spatially homogeneous PPP, that is $\Phi_{D}$ is a homogeneous PPPs with density $D\left[\mathrm{BS} / \mathrm{km}^{2}\right]$. Without loss of generality, we refer to $\mathrm{BS}_{0}$ as a typical BS and to $\mathrm{UE}_{0 k}$ as a typical UE. Particularly, they represent any BS and UE in the network by means of the translation invariance property of the homogeneous PPP. The $K$ UEs in each cell are assumed to be uniformly distributed within the Voronoi region around each BS. This means that the distance between $\mathrm{UE}_{l^{\prime} i}$ and $\mathrm{BS}_{l^{\prime}}$, denoted by $d_{l^{\prime} l^{\prime} i}[\mathrm{~km}]$, is distributed as $d_{l^{\prime} l^{\prime} i} \sim \operatorname{Rayleigh}\left(\frac{1}{\sqrt{2 \pi D}}\right)$. To model the LSF between $\mathrm{UE}_{l^{\prime} i}$ and $\mathrm{BS}_{l}$, we define $\beta_{l l^{\prime} i}=\omega^{-1} d_{l l^{\prime} i}^{-\alpha}$ where $\alpha$ is the pathloss exponent and $\omega$ is the pathloss at a reference distance of $1 \mathrm{~km}$. This parameter also accounts for propagation losses independent of the distances (e.g., wall penetration).

In cellular networks, the transmission power of UEs needs to be controlled in order to avoid that signals from UEs close to the BS overwhelm signals from UEs further away. This is particularly important in Massive MIMO where lowresolution analog-to-digital converters are expected to be used [34], [35]. Thus, we assume statistical channel inversion power control where the transmission power of data symbols is computed as $p_{l^{\prime} i}=\rho_{d} / \beta_{l^{\prime} l^{\prime} i}=\rho_{d} \omega d_{l^{\prime} l^{\prime} i}^{\alpha}$ and of pilot symbols as $q_{l^{\prime} i}=\rho_{p} / \beta_{l^{\prime} l^{\prime} i}=\rho_{p} \omega d_{l^{\prime} l^{\prime} i}^{\alpha}$. The design parameters $\rho_{d}$ and $\rho_{p}$ are used to control the average transmit power for data and pilot symbols, respectively. Moreover, we define $\rho$ as the total average transmission energy per symbol ${ }^{8}$ such that

\footnotetext{
${ }^{8}$ The average SNR per UEs is then given by $\mathrm{SNR}=\rho / \sigma^{2}$.
} 


$$
\begin{aligned}
\underline{\operatorname{SINR}}^{\mathrm{SP}}= & M(1-\Delta) /(\underbrace{\frac{M K}{\tau_{c}(\alpha-1)}\left(1-\frac{\Delta}{\tau_{c}}\right)+\frac{M K}{\tau_{c}} \frac{(1-\Delta) \alpha}{\Delta(\alpha-1)}}_{\text {Coherent interference }} \\
& +\underbrace{\frac{2(1-\Delta)}{\tau_{c}}\left(1+\frac{K}{\tau_{c}(\alpha-1)}\right)+\frac{K(1-\Delta)^{2} \alpha}{\tau_{c}^{2} \Delta(\alpha-1)}+\frac{K^{2}}{\tau_{c} \Delta(\alpha-1)}+\left(1+\frac{K}{\tau_{c} \Delta}\left(\frac{2}{(\alpha-2)}+(1-\Delta)\right)+\frac{\sigma^{2}}{\Delta \rho \tau_{c}}\right)\left(\frac{K \alpha}{\alpha-2}+\frac{\sigma^{2}}{\rho}\right)}_{\text {Non-coherent interference and noise }})
\end{aligned}
$$

$$
\begin{aligned}
\underline{\operatorname{SINR}}^{\mathrm{SP}-\mathrm{UB}}= & M(1-\Delta) /(\underbrace{\frac{M K(1-\Delta)}{\tau_{c}(\alpha-1)}+\frac{M K(1-\Delta)^{2} \alpha}{\tau_{c} \Delta(\alpha-1)}}_{\text {Coherent interference }} \\
& +\underbrace{\frac{K(1-\Delta)^{2} \alpha}{\tau_{c}^{2} \Delta(\alpha-1)}+\frac{K^{2}(1-\Delta)}{\tau_{c} \Delta(\alpha-1)}+\left(1+\frac{K}{\tau_{c} \Delta}\left(\frac{2}{(\alpha-2)}+(1-\Delta)\right)+\frac{\sigma^{2}}{\Delta \rho \tau_{c}}\right)\left(\frac{K(1-\Delta) \alpha}{\alpha-2}+\frac{\sigma^{2}}{\rho}\right)}_{\text {Non-coherent interference and noise }}) .
\end{aligned}
$$

$\rho_{d}=\rho_{p}=\rho$ with RP and $\rho_{d}+\rho_{p}=\rho$ with SP. We define the proportion between pilot and data power with SP as $\Delta \in[0,1]$ such that $\rho_{p}=\Delta \rho$ and $\rho_{d}=(1-\Delta) \rho$.

By introducing the aforementioned definitions of transmission powers and LSF coefficients, the achievable rates with RP and SP can be computed in terms of the distances between UEs and BSs. To get insights into the influence of design parameters such as the number of BS antennas $M$, the number of UEs per BS $K$, length of pilot sequences $\tau_{p}$ with RP and system parameters such as the size of the coherence block $\tau_{c}$, we evaluate the performance for different realizations of the UE positions. In particular, we calculate an expected value of $\mathrm{R}_{0 k}^{\mathrm{RP}}$ and $\mathrm{R}_{0 k}^{\mathrm{SP}}$ with respect to the distances $d_{l l^{\prime} i} \forall l, l^{\prime} \in \Phi_{D}$ and $i \in\{1, \ldots, K\}$. Following the same approach as in [8], a closed-form lower bound on the achievable rates can be computed as shown in the following theorem.

Theorem 2. A lower bound on the average ergodic achievable rate of the typical $\mathrm{UE}_{0 k}$ with respect to the UE positions when considering statistical channel inversion power control is with $R P$ given by

$$
\begin{aligned}
\underline{\mathrm{R}}^{\mathrm{RP}} & =B_{\mathrm{W}}\left(1-\frac{\tau_{p}}{\tau_{c}}\right) \log _{2}\left(1+\underline{\mathrm{SINR}}^{\mathrm{RP}}\right) \\
\underline{\mathrm{SINR}}^{\mathrm{RP}} & =\frac{M}{\frac{M K}{\tau_{p}(\alpha-1)}+\frac{K^{2}}{\tau_{p}(\alpha-1)}+\left(1+\frac{K}{\tau_{p}} \frac{2}{\alpha-2}+\frac{\sigma^{2}}{\rho \tau_{p}}\right)\left(\frac{\alpha K}{\alpha-2}+\frac{\sigma^{2}}{\rho}\right)}
\end{aligned}
$$

where $\rho_{d}=\rho_{p}=\rho$. With $S P$, it is given by

$$
\underline{\mathrm{R}}^{\mathrm{SP}}=B_{\mathrm{W}} \log _{2}\left(1+\underline{\mathrm{SINR}}^{\mathrm{SP}}\right)
$$

where $\underline{\mathrm{SINR}}^{\mathrm{SP}}$ is given in (34) at the top of the page.

By subtracting the pilot symbols perfectly from the data estimates, an upper bound on the effective SINR with SP is $\underline{\text { SINR }}^{\mathrm{SP}} \leq \underline{\mathrm{SINR}}^{\mathrm{SP}-\mathrm{UB}}$, shown in (35) at the top of the page. For both SP and RP, $\rho$ is the average transmission energy per symbol.
Proof: It follows from applying Jensen's inequality to the achievable rate as

$$
\mathbb{E}\left\{\log _{2}\left(1+\operatorname{SINR}_{0 k}\right)\right\} \geq \log _{2}\left(1+1 / \mathbb{E}\left\{\operatorname{SINR}_{0 k}^{-1}\right\}\right)
$$

where $\mathrm{SINR}_{0 k}$ represents the SINR of $\mathrm{UE}_{0 k}$ with either RP or SP. We then compute the moments of SINR ${ }_{0 k}^{-1}$. Notice that the expectation with respect to the distances results in an SINR expression independent of the UE index " $0, k$ ". See Appendix $\mathrm{C}$ for details on calculating $\mathbb{E}\left\{\mathrm{SINR}_{0 k}^{-1}\right\}$.

The lower bounds on the achievable rates with both RP and SP are increasing with $M$ and decreasing with $K$, which means that in order to serve more UEs with the same rates we need to increase the number of BS antennas. With RP, we can see that $\underline{\mathrm{SINR}}^{\mathrm{RP}}$ increases with $\tau_{p}$. However, the pre-log factor $\left(1-\tau_{p} / \tau_{c}\right)$ decreases with $\tau_{p}$. This means that the rate $\underline{\mathrm{R}}^{\mathrm{RP}}$ is a unimodal function of $\tau_{p}$ which can be maximized with bisection search algorithms. This result is in line with Corollary 3. If $\tau_{c}$ increases, then the pre-log factor $1-\tau_{p} / \tau_{c}$ increases as well since $\tau_{p} \in\left[K, \tau_{c}\right]$. This means that, by optimizing $\underline{\mathrm{R}}^{\mathrm{RP}}$ with respect to $\tau_{p}$, the maximum rate with $\mathrm{RP}$ increases with $\tau_{c}$. With $\mathrm{SP}$, the achievable rate $\underline{\mathrm{R}}^{\mathrm{SP}}$ also increases with $\tau_{c}$.

Notice that the closed-form expressions found in Theorem 2 do not require heavy numerical simulations and can give powerful insights into the data rates of practical network deployments.

\section{A. Energy Efficiency Modeling}

The closed-form achievable rates provided above to measure the SE of the network, allow us to provide analytical expressions for the EE, measured in [bit/Joule], with RP and SP. We consider the effect of transmission and circuit power consumption following the model in [7]. We define the EE as the ratio between the average sum data rate per unit area and 


\begin{tabular}{cccc}
\hline Param. & $M=100$ & $M=300$ & $M=500$ \\
\hline$\tau_{p}$ Opt. & 39 & 42 & 44 \\
\hline$\Delta$ no sub. & 0.36 & 0.45 & 0.5 \\
\hline$\Delta$ perf sub. & 0.6 & 0.7 & 0.75 \\
\hline$\Delta$ est. sub. & 0.47 & 0.5 & 0.53 \\
\hline
\end{tabular}

(a) Optimized parameters for $\rho=\sigma^{2} / 4$ ( $\mathrm{SNR}=-6 \mathrm{~dB}$ ), $K=10$ and $\tau_{c}=200$

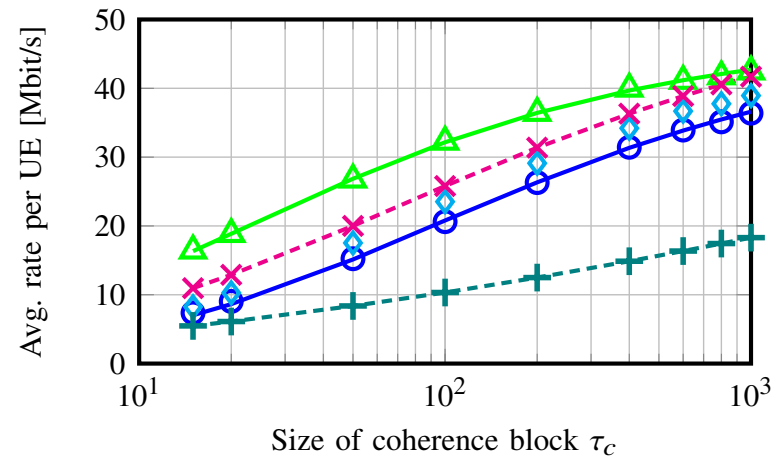

(c) Achievable rate per UE for $\rho=\sigma^{2} / 4(\mathrm{SNR}=-6 \mathrm{~dB})$, $M=100$ and $K=10$.

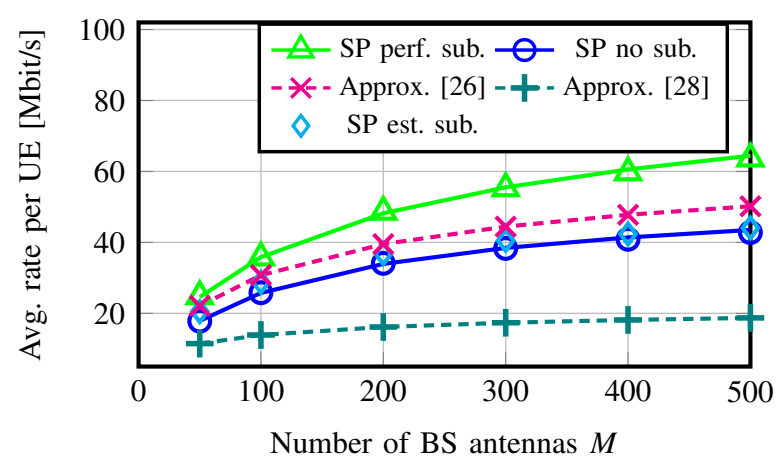

(b) Achievable rate per UE for $\rho=\sigma^{2} / 4(\mathrm{SNR}=-6 \mathrm{~dB})$, $\tau_{c}=200$ and $K=10$

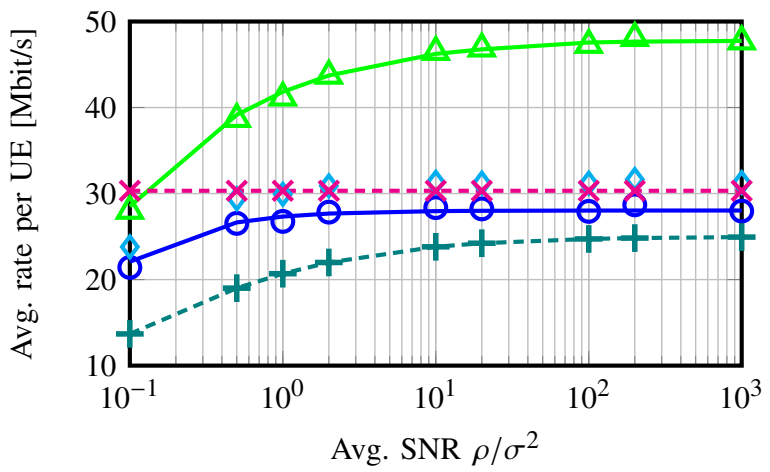

(d) Achievable rate per UE for $M=100, \tau_{c}=200$ and $K=10$.

Fig. 2: Optimized parameters and SP achievable rates versus $M, \tau_{c}$ and $\rho / \sigma^{2}$. The solid lines correspond to the closed-form expressions in Theorem 1 and Corollary 1, the triangle, diamond, and circle markers correspond to MC simulations over the SSF. All results are averaged over the LSF.

the average power consumption per unit area. This yields

$$
\begin{aligned}
\mathrm{EE} & =\frac{\mathbb{E}\left\{\sum_{k=1}^{K} \mathrm{R}_{0 k}\right\} D}{\mathrm{P}_{\mathrm{BS}} D} \\
& =\frac{\mathbb{E}\left\{\mathrm{R}_{0 k}\right\} K}{\mathrm{P}_{\mathrm{TX}}+C_{0}+C_{1} K+\mathcal{D}_{0} M+\mathrm{P}_{\mathrm{LP}}+\mathrm{P}_{\mathrm{CE}}+\mathcal{A} \mathbb{E}\left\{\mathrm{R}_{0 k}\right\} K}
\end{aligned}
$$

where $R_{0 k}$ is the achievable rate defined in (16) with RP and (24) with SP, and $\mathrm{P}_{\mathrm{BS}}$ is the power consumption per BS, which accounts for the transmission power and circuit power consumption (see the denominator of the second equality of (39)). Recall that $D$ is the density of BSs per unit area in $\left[\mathrm{BS} / \mathrm{km}^{2}\right]$. Note that $\mathbb{E}\left\{\mathrm{R}_{0 k}\right\}$ can be replaced by its lower bound in Theorem 2. By using Lemma 5 in Appendix D, the average transmission power is computed as

$$
\mathrm{P}_{\mathrm{TX}}=\frac{B_{\mathrm{W}}}{\eta} K \mathbb{E}\left\{p_{0 k}\right\}=\frac{B_{\mathrm{W}}}{\eta} K \rho \omega \frac{\Gamma(\alpha / 2+1)}{(\pi D)^{\alpha / 2}}
$$

where $\eta \in(0,1]$ denotes the efficiency of the power amplifier. The parameter $C_{0}$ accounts for fixed power consumption (e.g., site cooling), $C_{1}$ and $\mathcal{D}_{0}$ are the power consumed per transceiver chain of the UE and BS, respectively. The power consumption that depends on the data rates (e.g., coding, decoding, backhaul, etc.) is accounted in $\mathcal{A}$. The power consumption for linear processing and channel estimation is denoted by $\mathrm{P}_{\mathrm{LP}}$ and $\mathrm{P}_{\mathrm{CE}}$, respectively. To calculate $\mathrm{P}_{\mathrm{LP}}$ and
$\mathrm{P}_{\mathrm{CE}}$, we find a first-order approximation of the computational complexity (i.e., number of floating point operations per second (flops)), based on the number of complex multiplications in linear algebra operations, and then multiply it by the computational efficiency of current microprocessors denoted by $L[$ flops/W]. Then, we have

$$
\mathrm{P}_{\mathrm{LP}}+\mathrm{P}_{\mathrm{CE}}= \begin{cases}\frac{B_{\mathrm{W}}}{L} M K & \text { with RP } \\ 2 \frac{B_{\mathrm{W}}}{L} M K & \text { with SP. }\end{cases}
$$

The combined power consumption for linear processing and channel estimation is doubled by SP as compared to RP. This occurs because with SP we estimate the channel and detect the data for all symbols in the coherence block, whereas with $\mathrm{RP}$ we only estimate the channel in $\tau_{p}$ symbols and detect the data in $\tau_{c}-\tau_{p}$ symbols. Given that the computational efficiency of modern microprocessors is continuously increasing, a factor of two does not add a significant weight into the total power consumption with SP when compared to RP. Thus, the difference between RP and SP in terms of EE is going to be mainly determined by the rate performance. Comparisons are made in the next section.

\section{NUMERICAL RESULTS}

Monte Carlo (MC) simulations are used to compare RP and SP, and to validate the theoretical results of Sections IV, 


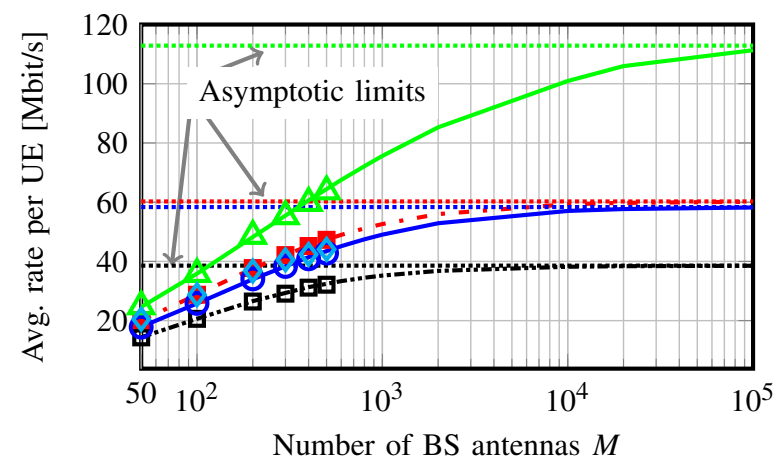

(a) Achievable rate per UE for $\rho=\sigma^{2} / 4(\mathrm{SNR}=-6 \mathrm{~dB})$, $\tau_{c}=200$ and $K=10$.

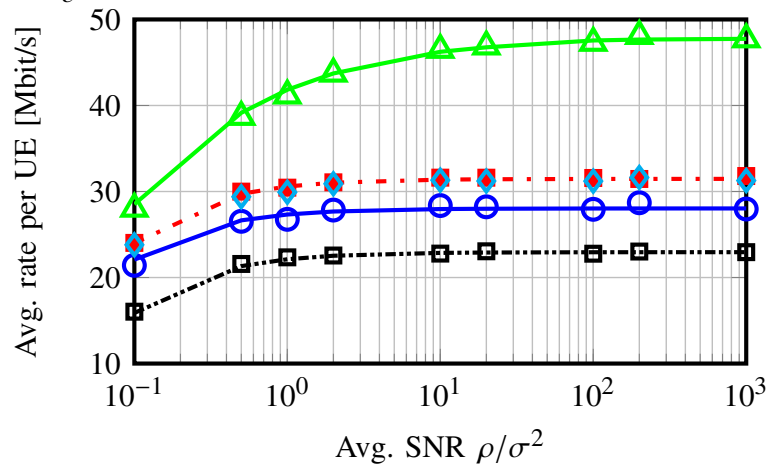

(c) Achievable rate per UE for $M=100, \tau_{c}=200$ and $K=10$.



(b) Achievable rate per UE for $\rho=\sigma^{2} / 4$ (SNR $\left.=-6 \mathrm{~dB}\right)$, $M=100$ and $K=10$.

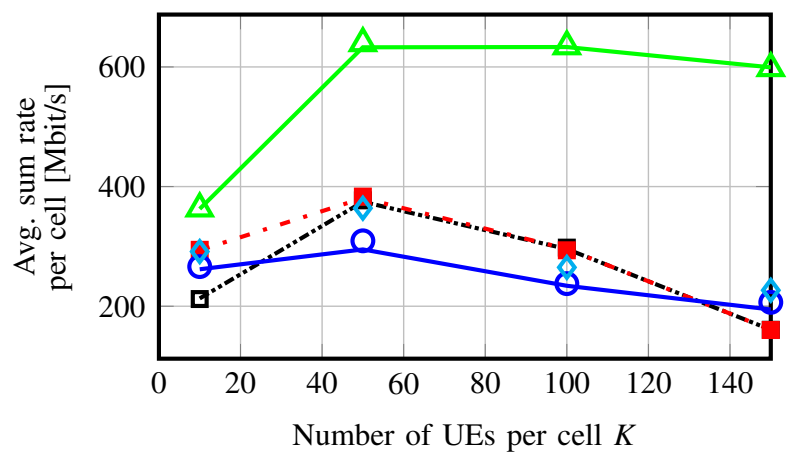

(d) Achievable sum rate per cell for $\rho=\sigma^{2} / 4(\mathrm{SNR}=-6 \mathrm{~dB})$, $M=100$ and $\tau_{c}=200$

Fig. 3: Achievable rates versus $M, \tau_{c}, \rho / \sigma^{2}$ and $K$. The lines correspond to the closed-form expressions in Lemma 3, Theorem 1 and Corollary 1. The markers correspond to MC simulations over the SSF. All results are averaged over the LSF.

TABLE II: Simulation Parameters

\begin{tabular}{c|c|c} 
Parameter & Symbol & Value \\
\hline Pathloss exponent & $\alpha$ & 3.76 \\
Fixed propagation loss $(1 \mathrm{~km})$ & $\omega$ & $130[\mathrm{~dB}]$ \\
System bandwidth & $B_{\mathrm{W}}$ & $20[\mathrm{MHz}]$ \\
Power amplifier efficiency & $\eta$ & 0.39 \\
Static power consumption & $C_{0}$ & $10[\mathrm{~W}]$ \\
Circuit power per active UE & $C_{1}$ & $0.1[\mathrm{~W}]$ \\
Circuit power per BS antenna & $\mathcal{D}_{0}$ & $0.1[\mathrm{~W}]$ \\
Power const. related to data rates & $\mathcal{A} B_{\mathrm{W}}$ & $2.3 \times 10^{-2}[\mathrm{~W} / \mathrm{bit}]$ \\
Computational efficiency & $L$ & $12.8[\mathrm{Gflops} / \mathrm{W}]$ \\
Noise power & $\sigma^{2} B_{\mathrm{W}}$ & $10^{-13}[\mathrm{~W}]$ \\
\hline
\end{tabular}

V, and VI. ${ }^{9}$ We simulate a homogeneous PPP with density $D=100\left[\mathrm{BS} / \mathrm{km}^{2}\right]$ in an squared area of side length $L_{\mathrm{SQ}}$ [km] with an average of $N_{a v}=D L_{\mathrm{SQ}}^{2}=50$ BSs. To avoid edge effects, we implement the wrap around technique where we replicate the original square 8 times and place the copies around itself. Table II summarizes the simulation parameters which are based on [7], [8] and references therein. We evaluate the performance of achievable rates and EE with MRC for the following methods:

- RP with pilot length equal to the number of users per BS,

\footnotetext{
${ }^{9}$ The simulation results can be reproduced with the code found at https: //github.com/emilbjornson/UL-superimposed-pilots
}

i.e., $\tau_{p}=K$;

- $\mathrm{RP}$ with optimal pilot length to maximize $\mathrm{R}_{0 k}^{\mathrm{RP}}$;

- SP as in Theorem 1 (denoted as "SP no sub., i.e., no pilot subtraction), Corollary 1 (denoted as "SP perf. sub.",i.e., perfect pilot subtraction) and Theorem 2;

- SP when we subtract the estimated received pilot symbols from the data estimate $\left[\hat{\mathbf{s}}_{0 k}\right]_{j}$ in (22), denoted as "SP est. sub." which stands for estimated pilot subtraction;

- SP with the approximated results found in [26], [28] denoted as "Approx. [26]" and "Approx. [28]" respectively.

Note that in all figures the proportion $\Delta$ between pilot and data power with SP is optimized to maximize the data rates in each LSF realization.

Fig. 2a shows a table with the average $\tau_{p}$ and $\Delta$ values that maximize the data rates. The optimal $\tau_{p}$ covers approximately $20 \%$ of the coherence block and it increases with $M$ to counteract the effect of pilot contamination. With SP, the optimal $\Delta$ also increases with $M$; which is in line with the results from [26]. From the results with and without pilot subtraction, we can see that the optimal $\Delta$ seeks to balance the interference from pilot symbols and the quality of channel estimation. The rest of the graphs in Fig. 2 show the average data rate per UE versus the number of BS antennas, size of the coherence block, and average SNR. The MC results confirm the validity of the closed-form expressions found in Theorem 1 and Corollary 1. It can be seen that there 


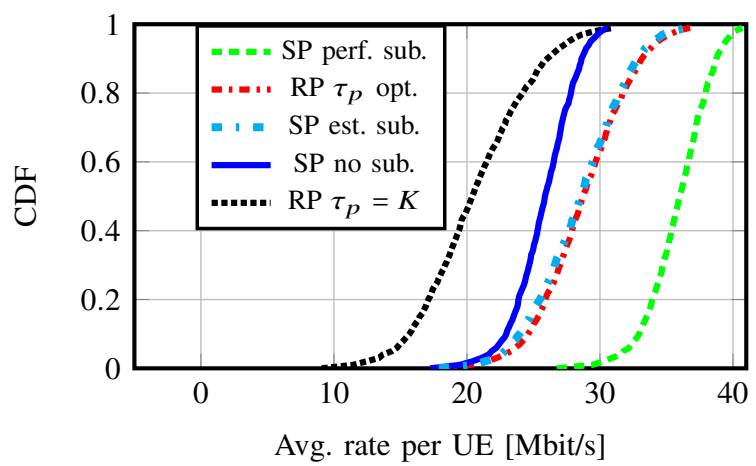

(a) $\mathrm{CDF}$ of achievable rate per UE for $M=100, \rho=\sigma^{2} / 4$ $(\mathrm{SNR}=-6 \mathrm{~dB}), \tau_{c}=200$ and $K=10$.

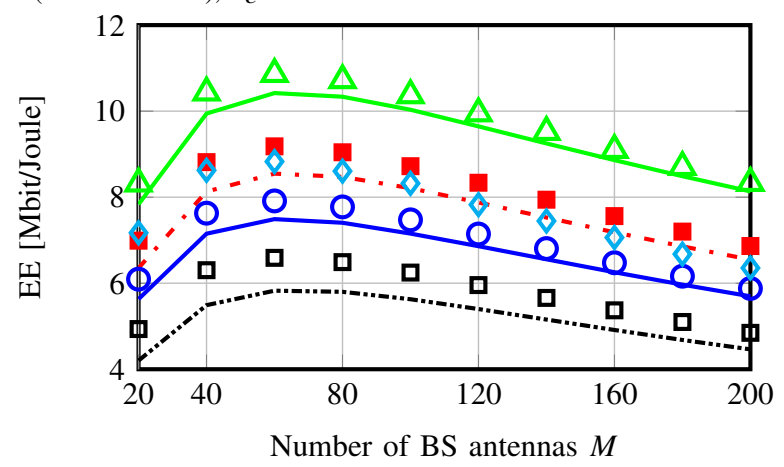

(c) EE for $\rho=\sigma^{2} / 4(\mathrm{SNR}=-6 \mathrm{~dB}), \tau_{c}=200$ and $K=10$.

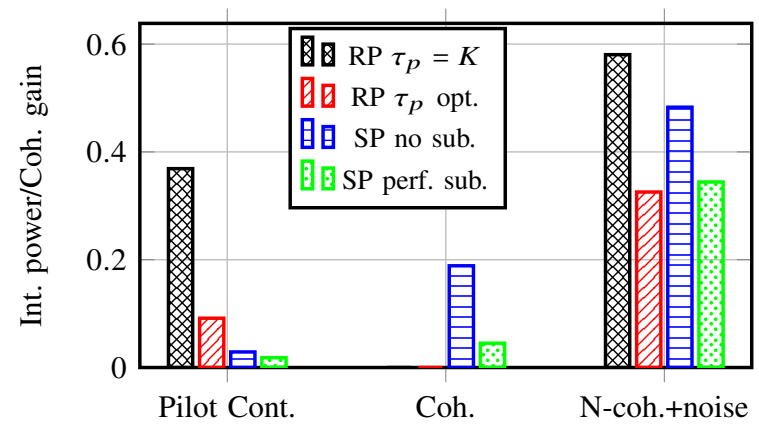

(b) Interference sources for $M=100, \rho=\sigma^{2} / 4$ $(\mathrm{SNR}=-6 \mathrm{~dB}), \tau_{c}=200$ and $K=10$.

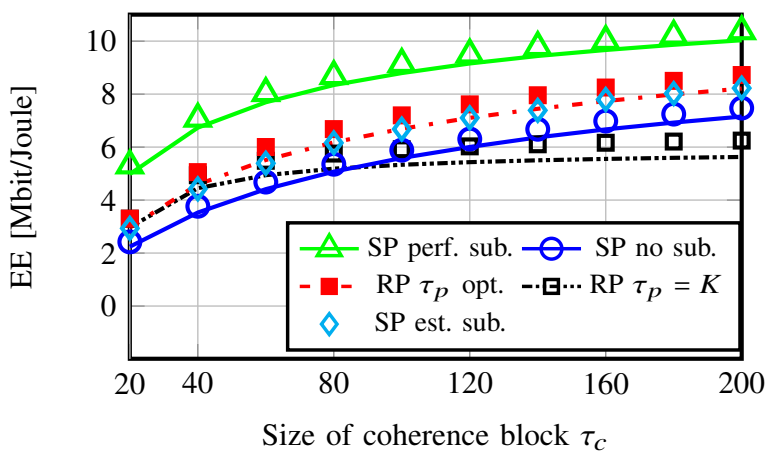

(d) EE for $\rho=\sigma^{2} / 4(\mathrm{SNR}=-6 \mathrm{~dB}), M=100$ and $K=10$.

Fig. 4: CDF of achievable rates, interference sources, and EE versus $M$ and $\tau_{c}$. In Figs. $4 \mathrm{c}$ and $4 \mathrm{~d}$, the markers are based on the closed-form expressions in Lemma 3, Theorem 1 and Corollary 1, averaged over the LSF. The lines are based on the closed-form expressions in Theorem 2.

is a large gap between the results with no pilot subtraction and perfect pilot subtraction. However, the data rates with estimated pilot subtraction are closer to the data rates with no pilot subtraction, which is due to the cross products that arise from the correlation between channel estimates and data symbols. The approximation [26] is found within the results derived in Theorem 1 and Corollary 1, which makes it a good approximation of the performance. However, since the impact of noise is neglected in [26], the approximation becomes less accurate in very low SNR scenarios. On the other hand, the approximation in [28] greatly underestimates the performance with SP.

Fig. 3 depicts the achievable rates per UE versus the number of BS antennas, coherence block size, and average SNR along with the sum rate per cell versus the number UEs per cell. In general, we can see that SP outperforms RP with $\tau_{p}=K$ for most cases when $\tau_{c}$ is long enough to reduce pilot contamination. Otherwise, the data rates with RP, including the results when $\tau_{p}$ is optimized, provide comparable performance to that of SP with estimated pilot subtraction. When subtracting the pilot symbols perfectly, the data rates with SP provide the best performance, but it might be hard to achieve this in practice. In Fig. 3a, the asymptotic limits derived in Corollary 2 are shown. We can see that more than $10^{4} \mathrm{BS}$ antennas are needed to converge to the limits and the relative differences among the methods vary between the finite $M$ and $M \rightarrow \infty$.
Fig. 4a depicts the cumulative distribution function (CDF) of the achievable rates per UE for different realizations of LSF. We can see that the rate distribution does not show any large difference between the evaluated methods. Fig. 4b shows the strength of the interference sources with respect to the coherent gain (all terms are defined in Table I). We see that with SP, there is a reduction of the pilot contamination contributions with respect to RP. At the same time, however, additional coherent interference appears from data transmission and, in the case of SP without pilot subtraction, that is substantial. It is important to note that the overall impact of coherent interference for $M=100$ and $K=10$ is lower than the impact of non-coherent interference with both RP and SP. This suggests that, in practical dense deployments, Massive MIMO systems may not be limited by coherent interference. Fig. $4 \mathrm{c}$ and Fig. $4 \mathrm{~d}$ depict the EE in terms of the number of BS antennas and size of the coherence block respectively. We see that the closed-form lower bounds found in Theorem 2 follow the same trend as the MC simulations. In general, we can see a similar trend as in previous results, SP outperforms RP when $\tau_{p}=K$, however when we optimize $\tau_{p}$ we find that RP provides comparable EE than SP with estimated pilot subtraction. The highest EE is found with SP when pilot symbols are subtracted perfectly. 


\section{CONCLUSIONS}

In this paper, we derived the first rigorous achievable rate expression for a multicell Massive MIMO network with SP. We analytically and numerically compared RP and SP in a practical multicell deployment. By examining the contribution of different sources of interference, we observed that SP is able to reduce pilot contamination at the expense of incorporating further coherent and non-coherent interference that limits the system performance. The results showed that, by optimizing the pilot length with RP, the average SE and EE are comparable to SP when estimated pilot subtraction is used. On the other hand, when the pilot symbols are subtracted perfectly with SP, the SE and EE are the highest, which indicates that there is room for improvement-iterative decoding algorithms might be able to bridge this gap. When analyzing the large number of BS antennas regime, we encountered that asymptotic results do not convey accurate results to gain insights into the behavior of practical deployments. Moreover, it is worth to stress that in practical deployments the effect of coherent interference, such as pilot contamination, can be less influential than noncoherent inter-cell on the SE of UEs.

The use of SP has the potential to provide better performance by using other signal processing schemes like zeroforcing, multicell MMSE decoding, as in [12] or iterative decoding algorithms as in [26], [28]. Moreover, channel estimation can be improved by the use of (semi-)blind estimation methods such as those in [16]-[18]. However, it is not clear whether the benefit of using such schemes would compensate for the increased computational complexity. All this study is left for future work.

\section{APPENDIX A}

\section{PROOF OF THEOREM 1}

Since the channels are circularly symmetric complex Gaussian random vectors, the channel gain uncertainty term (i.e., the first term in the denominator in (26)) can be computed as (e.g., [11, Appendix A])

$$
\frac{\mathbb{E}\left\{\left\|\mathbf{h}_{00 k}\right\|^{4}\right\}-\left|\mathbb{E}\left\{\left\|\mathbf{h}_{00 k}\right\|^{2}\right\}\right|^{2}}{M \beta_{00 k}}=\frac{M(M+1) \beta_{00 k}^{2}-M^{2} \beta_{00 k}^{2}}{M \beta_{00 k}}=\beta_{00 k} .
$$

To calculate the variance of the rest of the effective noise, we first condition on an arbitrary realization of $\chi_{l^{\prime} i}^{\mathrm{SP}} \forall l^{\prime} \in \Phi_{D}$, $i \in\{1, \ldots, K\}$ and then compute the expectation over $\chi_{l^{\prime} i}^{\mathrm{SP}}$ as

$$
\begin{aligned}
\operatorname{Var}\left(n_{\text {eff }}\right) & =\mathbb{E}\left\{\left|n_{\text {eff }}-\mathbb{E}\left\{n_{\text {eff }}\right\}\right|^{2}\right\} \\
& =\mathbb{E}\left\{\mathbb{E}\left\{\left|n_{\text {eff }}\right|^{2} \mid \chi_{l^{\prime} i}^{\mathrm{SP}}\right\}\right\}-\left|\mathbb{E}\left\{\mathbb{E}\left\{n_{\text {eff }} \mid \chi_{l^{\prime} i}^{\mathrm{SP}}\right\}\right\}\right|^{2} .
\end{aligned}
$$

To proceed further, the following lemma is needed.

Lemma 4. ( $[11$, Appendix A]) Consider two independent random vectors distributed as $\mathbf{x} \sim \mathcal{C N}\left(\mathbf{0}, \sigma_{x}^{2} \mathbf{I}_{M}\right)$ and $\mathbf{y} \sim \mathcal{C N}\left(\mathbf{0}, \sigma_{y}^{2} \mathbf{I}_{M}\right)$, then we have the following results:

$$
\begin{aligned}
& \mathbb{E}\left\{(\mathbf{x}+\mathbf{y})^{H} \mathbf{x}\right\}=\mathbb{E}\left\{\|\mathbf{x}\|^{2}\right\}=M \sigma_{x}^{2} \\
& \mathbb{E}\left\{\left|(\mathbf{x}+\mathbf{y})^{H} \mathbf{x}\right|^{2}\right\}=M(M+1) \sigma_{x}^{4}+M \sigma_{x}^{2} \sigma_{y}^{2} .
\end{aligned}
$$

By applying Lemma 4 and the result from (13) to (44), we have that

$$
\begin{aligned}
& \mathbb{E}\left\{\mathbb{E}\left\{n_{e f f} \mid \chi_{l^{\prime} i}^{\mathrm{SP}}\right\}\right\}=\sqrt{\frac{M}{q_{0 k} \beta_{00 k}}} \mathbb{E}\left\{\left(\frac{p_{0 k} \beta_{00 k}}{\tau_{c}}\left[\boldsymbol{\varphi}_{0 k}\right]_{j}\right.\right. \\
& \left.\left.+\sum_{l^{\prime} \in \Phi_{D}} \sum_{i=1}^{K}\left(\chi_{l^{\prime} i}^{\mathrm{SP}} q_{l^{\prime} i}\left[\boldsymbol{\varphi}_{l^{\prime} i}\right]_{j}+\frac{\xi_{l^{\prime} i} p_{l^{\prime} i}}{\tau_{c}}\left[\boldsymbol{\varphi}_{0 k}\right]_{j}\right) \beta_{0 l^{\prime} i}+\frac{\sigma^{2}\left[\boldsymbol{\varphi}_{0 k}\right]_{j}}{\tau_{c}}\right)\right\} \\
& =\sqrt{\frac{M}{q_{0 k} \beta_{00 k}}}\left(q_{0 k} \beta_{00 k}+\frac{1}{\tau_{c}} \sum_{l^{\prime} \in \Psi_{D}} \sum_{i=1}^{K} q_{l^{\prime} i} \beta_{0 l^{\prime} i}\right. \\
& \left.+\frac{1}{\tau_{c}} \sum_{l^{\prime} \in \Phi_{D}} \sum_{i=1}^{K} p_{l^{\prime} i} \beta_{0 l^{\prime} i}+\frac{\sigma^{2}}{\tau_{c}}\right)\left[\boldsymbol{\varphi}_{0 k}\right]_{j}
\end{aligned}
$$

and the expression for $\mathbb{E}\left\{\mathbb{E}\left\{\left|n_{\text {eff }}\right|^{2} \mid \chi_{l^{\prime} i}^{\mathrm{SP}}\right\}\right\}$ is in (51) in the page after the next. By combining (47) with (51), we have that

$$
\begin{gathered}
\operatorname{Var}\left(n_{e f f}\right)=\frac{M}{q_{0 k} \beta_{00 k}}\left(\frac{1}{\tau_{c}} \sum_{l^{\prime} \in \Psi_{D}} \sum_{i=1}^{K}\left(p_{l^{\prime} i}+\left(1-\frac{1}{\tau_{c}}\right) q_{l^{\prime} i}\right) q_{l^{\prime} i} \beta_{0 l^{\prime} i}^{2}\right. \\
\left.+\frac{1}{\tau_{c}} \sum_{l^{\prime} \in \Phi_{D}} \sum_{i=1}^{K}\left(q_{l^{\prime} i}+p_{l^{\prime} i}\right) p_{l^{\prime} i} \beta_{0 l^{\prime} i}^{2}\right) \\
+\frac{1}{\tau_{c} q_{0 k} \beta_{00 k}}\left(\frac{2}{\tau_{c}} \sum_{l^{\prime} \in \Psi_{D}} \sum_{i=1}^{K} p_{l^{\prime} i} q_{l^{\prime} i} \beta_{0 l^{\prime} i}^{2}+\frac{1}{\tau_{c}} \sum_{l^{\prime} \in \Phi_{D}} \sum_{i=1}^{K} p_{l^{\prime} i}^{2} \beta_{0 l^{\prime} i}^{2}\right) \\
+p_{0 k} \beta_{00 k}\left(-1+\frac{2}{\tau_{c}}\right)+\frac{1}{\gamma_{0 k}^{\mathrm{SP}}}\left(\sum_{l^{\prime} \in \Phi_{D}} \sum_{i=1}^{K}\left(q_{l^{\prime} i}+p_{l^{\prime} i}\right) \beta_{0 l^{\prime} i}+\sigma^{2}\right)
\end{gathered}
$$

and by combining (25) and (26) with (42) and (48), the proof is complete.

\section{APPENDIX B}

\section{PROOF OF COROLLARY 3}

By studying the first and second derivative of $\mathrm{R}_{0 k}^{\mathrm{A}-\mathrm{RP}}$ with respect to $\zeta$, we have

$$
\begin{aligned}
\frac{\partial \mathrm{R}_{0 k}^{\mathrm{A}-\mathrm{RP}}(\zeta)}{\partial \zeta} & =\left(-\frac{\ln \left(1+\zeta \mathrm{SIR}_{\mathrm{RP}}\right)}{\ln (2)}+\frac{(1-\zeta) \mathrm{SIR}_{\mathrm{RP}}}{\ln (2)\left(1+\zeta \mathrm{SIR}_{\mathrm{RP}}\right)}\right) B_{\mathrm{W}} \\
\frac{\partial^{2} \mathrm{R}_{0 k}^{\mathrm{A}-\mathrm{RP}}(\zeta)}{\partial \zeta^{2}} & =-\frac{\left(2+(1+\zeta) \mathrm{SIR}_{\mathrm{RP}}\right)}{\mathrm{SIR}_{\mathrm{RP}} \ln (2)\left(1+\zeta \mathrm{SIR}_{\mathrm{RP}}\right)^{2}} B_{\mathrm{W}}<0 .
\end{aligned}
$$

We can see that $\mathrm{R}_{0 k}^{\mathrm{A}-\mathrm{RP}}(\zeta)$ is a concave function and $\mathrm{R}_{0 k}^{\mathrm{A}-\mathrm{RP}}(0)=\mathrm{R}_{0 k}^{\mathrm{A}-\mathrm{RP}}(1)=0$. Thus, considering that $\mathrm{SIR}_{\mathrm{RP}}>0$, the maximum point of $\mathrm{R}_{0 k}^{\mathrm{A}-\mathrm{RP}}(\zeta)$ is obtained when its derivative is zero and it is found at $\zeta^{\max }$ shown in (33). This concludes the proof.

\section{APPENDIX C \\ PROOF OF THEOREM 2}

By introducing the definitions of $q_{l^{\prime} i}=p_{l^{\prime} i}=\rho / \beta_{l^{\prime} l^{\prime} i}$ with RP, $q_{l^{\prime} i}=\Delta \rho / \beta_{l^{\prime} l^{\prime} i}$ and $p_{l^{\prime} i}=(1-\Delta) \rho / \beta_{l^{\prime} l^{\prime} i}$ with SP and, $\beta_{l^{\prime} l^{\prime} i}=\omega^{-1} d_{l^{\prime} l^{\prime} i}^{-\alpha}$, into (18), (27) and (28) we have the expressions in (58), (59), and (60) shown in the page after the next. 


$$
\begin{aligned}
& \mathbb{E}\left\{\mathbb{E}\left\{\left|n_{\text {eff }}\right|^{2} \mid \chi_{l^{\prime} i}^{\mathrm{SP}}\right\}\right\}=\mathbb{E}\left\{\mathbb { E } \left\{p_{0 k}\left|\overline{\mathbf{v}}_{00 k}^{H} \mathbf{h}_{00 k}\left[\mathbf{s}_{0 k}\right]_{j}\right|^{2}+\left|\mathbf{v}_{00 k}^{H} \mathbf{n}_{0 j}\right|^{2}\right.\right. \\
& \left.+\left.\left|\sum_{l^{\prime} \in \Phi_{D}} \sum_{i=1}^{K}\left(\sqrt{q_{l^{\prime} i}}\left[\varphi_{l^{\prime} i}\right]_{j}+\xi_{l^{\prime} i} \sqrt{p_{l^{\prime} i}}\left[\mathbf{s}_{l^{\prime} i}\right]_{j}\right) \mathbf{v}_{00 k}^{H} \mathbf{h}_{0 l^{\prime} i}\right|\right|^{2} \mid \chi_{l^{\prime} i}^{\mathrm{SP}}\right\} \\
& +2 \mathfrak{R}\left(\mathbb{E}\left\{\mathbf{v}_{00 k}^{H} \mathbf{n}_{0 j} \sqrt{p_{0 k}} \mathbf{h}_{00 k}^{H} \overline{\mathbf{v}}_{00 k}\left[\mathbf{s}_{0 k}\right]_{j}^{*} \mid \chi_{l^{\prime} i}^{\mathrm{SP}}\right\}\right) \\
& +2 \mathfrak{R}\left(\mathbb{E}\left\{\mathbf{v}_{00 k}^{H} \mathbf{n}_{0 j} \sum_{l^{\prime} \in \Phi_{D}} \sum_{i=1}^{K} \mathbf{h}_{0 l^{\prime} i}^{H} \mathbf{v}_{00 k}\left(\sqrt{q_{l^{\prime} i}}\left[\varphi_{l^{\prime} i}\right]_{j}^{*}+\xi_{l^{\prime} i} \sqrt{p_{l^{\prime} i}}\left[\mathbf{s}_{l^{\prime}} i\right]_{j}^{*}\right) \mid \chi_{l^{\prime} i}^{\mathrm{SP}}\right\}\right) \\
& \left.+2 \mathfrak{R}\left(\mathbb{E}\left\{\sqrt{p_{0 k}} \overline{\mathbf{v}}_{00 k}^{H} \mathbf{h}_{00 k}\left[\mathbf{s}_{0 k}\right]_{j} \sum_{l^{\prime} \in \Phi_{D}} \sum_{i=1}^{K} \mathbf{h}_{0 l^{\prime} i}^{H} \mathbf{v}_{00 k}\left(\sqrt{q_{l^{\prime}}}\left[\boldsymbol{\varphi}_{l^{\prime}}\right]_{j}^{*}+\xi_{l^{\prime} i} \sqrt{p_{l^{\prime} i}}\left[\mathbf{s}_{l^{\prime} i}\right]_{j}^{*}\right) \mid \chi_{l^{\prime} i}^{\mathrm{SP}}\right\}\right)\right\} \\
& =p_{0 k} \beta_{00 k}\left(\frac{1}{\gamma_{0 k}^{\mathrm{SP}}}-1\right)+\frac{M}{\tau_{c}} \frac{p_{0 k}}{q_{0 k}} p_{0 k} \beta_{00 k}+\frac{(M+1)}{\tau_{c}^{2}} \frac{p_{0 k}}{q_{0 k}} p_{0 k} \beta_{00 k}+\frac{\sigma^{2}}{\gamma_{0 k}^{\mathrm{SP}}}+M \frac{\sigma^{4}}{\tau_{c}^{2} q_{0 k} \beta_{00 k}} \\
& +\frac{M}{q_{0 k} \beta_{00 k}}\left(\left(q_{0 k} \beta_{00 k}+\frac{1}{\tau_{c}} \sum_{l^{\prime} \in \Psi_{D}} \sum_{i=1}^{K} q_{l^{\prime} i} \beta_{0 l^{\prime} i}+\sum_{l^{\prime} \in \Phi_{D}} \sum_{i=1}^{K} \xi_{l^{\prime} i} \frac{p_{l^{\prime} i}}{\tau_{c}} \beta_{0 l^{\prime} i}\right)^{2}+\frac{1}{\tau_{c}}\left(1-\frac{1}{\tau_{c}}\right) \sum_{l^{\prime} \in \Psi_{D}} \sum_{i=1}^{K} q_{l^{\prime} i}^{2} \beta_{0 l^{\prime} i}^{2}\right) \\
& +\frac{M}{q_{0 k} \beta_{00 k}}\left(\frac{1}{\tau_{c}} \sum_{l^{\prime} \in \Psi_{D}} \sum_{i=1}^{K} p_{l^{\prime} i} q_{l^{\prime} i} \beta_{0 l^{\prime} i}^{2}+\frac{1}{\tau_{c}} \sum_{l^{\prime} \in \Phi_{D}} \sum_{i=1}^{K}\left(q_{l^{\prime} i}+\xi_{l^{\prime} i} p_{l^{\prime} i}\right) p_{l^{\prime} i} \beta_{0 l^{\prime} i}^{2}\right) \\
& +\frac{1}{\tau_{c} q_{0 k} \beta_{00 k}}\left(\frac{2}{\tau_{c}} \sum_{l^{\prime} \in \Psi_{D}} \sum_{i=1}^{K} p_{l^{\prime} i} q_{l^{\prime} i} \beta_{0 l^{\prime} i}^{2}+\frac{1}{\tau_{c}} \sum_{l^{\prime} \in \Phi_{D}} \sum_{i=1}^{K} \xi_{l^{\prime} i} p_{l^{\prime} i}^{2} \beta_{0 l^{\prime} i}^{2}\right)+\frac{1}{\gamma_{0 k}^{\mathrm{SP}}}\left(\sum_{l^{\prime} \in \Phi_{D}} \sum_{i=1}^{K}\left(q_{l^{\prime} i}+\xi_{l^{\prime} i} p_{l^{\prime} i}\right) \beta_{0 l^{\prime} i}\right) \\
& +2 \frac{M \sigma^{2}}{\tau_{c}^{2}} \frac{p_{0 k}}{q_{0 k}}+2 \frac{M \sigma^{2}}{\tau_{c} q_{0 k} \beta_{00 k}}\left(q_{0 k} \beta_{00 k}+\frac{1}{\tau_{c}} \sum_{l^{\prime} \in \Psi_{D}} \sum_{i=1}^{K} q_{l^{\prime} i} \beta_{0 l^{\prime} i}+\sum_{l^{\prime} \in \Phi_{D}} \sum_{i=1}^{K} \xi_{l^{\prime} i} \frac{p_{l^{\prime} i}}{\tau_{c}} \beta_{0 l^{\prime} i}\right) \\
& +2\left(\frac{p_{0 k} \beta_{00 k}}{\tau_{c}}+\frac{M}{\tau_{c}} \frac{p_{0 k}}{q_{0 k}}\left(q_{0 k} \beta_{00 k}+\frac{1}{\tau_{c}} \sum_{l^{\prime} \in \Psi_{D}} \sum_{i=1}^{K} q_{l^{\prime} i} \beta_{0 l^{\prime} i}+\sum_{l^{\prime} \in \Phi_{D}} \sum_{i=1}^{K} \xi_{l^{\prime} i} \frac{p_{l^{\prime} i}}{\tau_{c}} \beta_{0 l^{\prime} i}\right)\right) \text {. }
\end{aligned}
$$

The expected value of the first term in $\mathrm{SINR}_{0 k}^{\mathrm{RP}-1}$ (first term in the denominator of (58)) with RP and the expected value of all terms except the last one in $\mathrm{SINR}_{0 k}^{\mathrm{SP}-1}$ and $\mathrm{SINR}_{0 k}^{\mathrm{SP}-\mathrm{UB}-1}$ (in the denominator of (59) and (60) respectively) with SP, are given by applying (55) in Lemma 6 of Appendix D. For the second term in $\mathrm{SINR}_{0 k}^{\mathrm{RP}-1}$ with $\mathrm{RP}$, and the last term in $\operatorname{SINR}_{0 k}^{\mathrm{SP}-1}$ and $\mathrm{SINR}_{0 k}^{\mathrm{SP}-\mathrm{UB}-1}$ with $\mathrm{SP}$, we can apply the following result

$$
\begin{aligned}
& \mathbb{E}\left\{\left(\mathrm{G}+\mathrm{T} \sum_{l^{\prime} \in \Psi_{D}} \sum_{i=1}^{K} \frac{d_{l^{\prime} l^{\prime}}^{\alpha}}{d_{0 l^{\prime} i}^{\alpha}}\right)\left(\mathrm{B}+\mathrm{J} \sum_{l^{\prime} \in \Psi_{D}} \sum_{i=1}^{K} \frac{d_{l^{\prime} l^{\prime} i}^{\alpha}}{d_{0 l^{\prime}}^{\alpha}}\right)\right\} \\
& =\mathrm{GB}+\mathrm{GJ} \sum_{i=1}^{K} \mathbb{E}\left\{\sum_{l^{\prime} \in \Psi_{D}} \frac{d_{l^{\prime} l^{\prime} i}^{\alpha}}{d_{0 l^{\prime} i}^{\alpha}}\right\}+\mathrm{BT} \sum_{i=1}^{K} \mathbb{E}\left\{\sum_{l^{\prime} \in \Psi_{D}} \frac{d_{l^{\prime} l^{\prime} i}^{\alpha}}{d_{0 l^{\prime} i}^{\alpha}}\right\} \\
& +\mathrm{JT} \sum_{i=1}^{K} \sum_{j=1}^{K} \mathbb{E}\left\{\left(\sum_{l^{\prime} \in \Psi_{D}} \frac{d_{l^{\prime} l^{\prime} i}^{\alpha}}{d_{0 l^{\prime} i}^{\alpha}}\right)\left(\sum_{l \in \Psi_{D}} \frac{d_{l l j}^{\alpha}}{d_{0 l j}^{\alpha}}\right)\right\} \\
& =\mathrm{GB}+\mathrm{GJ} \frac{2 K}{\alpha-2}+\mathrm{BT} \frac{2 K}{\alpha-2} \\
& +\mathrm{JT} \sum_{i=1}^{K} \sum_{j=1}^{K}\left(\mathbb{E}\left\{\sum_{l^{\prime} \in \Psi_{D}} \sum_{l \in \Psi_{D} \backslash\left\{l^{\prime}\right\}} \frac{d_{l^{\prime} l^{\prime} i}^{\alpha}}{d_{0 l^{\prime} i}^{\alpha}} \frac{d_{l l j}^{\alpha}}{d_{0 l j}^{\alpha}}\right\}+\mathbb{E}\left\{\sum_{l^{\prime} \in \Psi_{D}} \frac{d_{l^{\prime} l^{\prime} i}^{\alpha}}{d_{0 l^{\prime} i}^{\alpha}} \frac{d_{l^{\prime} l^{\prime} j}^{\alpha}}{d_{0 l^{\prime} j}^{\alpha}}\right\}\right)
\end{aligned}
$$

$$
\leq \mathrm{GB}+\mathrm{GJ} \frac{2 K}{\alpha-2}+\mathrm{BT} \frac{2 K}{\alpha-2}+\mathrm{JTK} K^{2}\left(\left(\frac{2}{\alpha-2}\right)^{2}+\frac{1}{\alpha-1}\right)
$$

which follows from Lemma 6, in Appendix D. For the case of RP we have

$$
\mathrm{G}=1+\frac{\sigma^{2}}{\rho \tau_{p}}, \quad \mathrm{~T}=\frac{1}{\tau_{p}}, \quad \mathrm{~B}=K+\frac{\sigma^{2}}{\rho}, \quad \mathrm{J}=1,
$$

and by combining (52) with (53) we obtain the last two terms in the denominator of $\underline{\operatorname{SINR}}^{\mathrm{RP}}$ in (37). In the case of SP, we have

$\mathrm{G}=1+\frac{K(1-\Delta)}{\Delta \tau_{c}}+\frac{\sigma^{2}}{\Delta \rho \tau_{c}}, \quad \mathrm{~T}=\frac{1}{\Delta \tau_{c}}, \quad \mathrm{~B}=K+\frac{\sigma^{2}}{\rho}, \quad \mathrm{J}=1$,

and by combining (52) with (54) the last two terms in the denominators of SINR ${ }^{S P}$ in (34) are obtained. For the case of SP with perfect pilot subtraction we have the same values as in (54) except for $\mathrm{B}=K(1-\Delta)+\frac{\sigma^{2}}{\rho}$ and $\mathrm{J}=1-\Delta$. Then by combining these values with (52) the last two terms in the denominator of $\underline{\text { SINR }}^{\text {SP-UB }}$ in (35) are found. Thus, the proof is concluded. 


$$
\begin{aligned}
& \operatorname{SINR}_{0 k}^{\mathrm{RP}}=\frac{M}{\frac{M}{\tau_{p}} \sum_{l^{\prime} \in \Psi_{D}} \sum_{i=1}^{K}\left(\frac{d_{l^{\prime} l^{\prime} i}^{\alpha}}{d_{0 l^{\prime} i}^{\alpha}}\right)^{2}+\left(1+\frac{1}{\tau_{p}} \sum_{l^{\prime} \in \Psi_{D}} \sum_{i=1}^{K} \frac{d_{l^{\prime} l^{\prime} i}^{\alpha}}{d_{0 l^{\prime} i}^{\alpha}}+\frac{\sigma^{2}}{\rho \tau_{p}}\right)\left(K+\sum_{l^{\prime} \in \Psi_{D}} \sum_{i=1}^{K} \frac{d_{l^{\prime} l^{\prime} i}^{\alpha}}{d_{0 l^{\prime} i}^{\alpha}}+\frac{\sigma^{2}}{\rho}\right)} \\
& \operatorname{SINR}_{0 k}^{\mathrm{SP}}=M(1-\Delta) /\left(\frac{M}{\tau_{c}}\left(1-\frac{\Delta}{\tau_{c}}\right) \sum_{l^{\prime} \in \Psi_{D}} \sum_{i=1}^{K}\left(\frac{d_{l^{\prime} l^{\prime} i}^{\alpha}}{d_{0 l^{\prime} i}^{\alpha}}\right)^{2}+\frac{M}{\tau_{c}} \frac{(1-\Delta)}{\Delta}\left(K+\sum_{l^{\prime} \in \Psi_{D}} \sum_{i=1}^{K}\left(\frac{d_{l^{\prime} l^{\prime} i}^{\alpha}}{d_{0 l^{\prime} i}^{\alpha}}\right)^{2}\right)+\frac{2}{\tau_{c}}(1-\Delta)+\frac{2(1-\Delta)}{\tau_{c}^{2}} \sum_{l^{\prime} \in \Psi_{D}} \sum_{i=1}^{K}\left(\frac{d_{l^{\prime} l^{\prime} i}^{\alpha}}{d_{0 l^{\prime} i}^{\alpha}}\right)^{2}\right. \\
& \left.+\frac{(1-\Delta)^{2}}{\tau_{c}^{2} \Delta}\left(K+\sum_{l^{\prime} \in \Psi_{D}} \sum_{i=1}^{K}\left(\frac{d_{l^{\prime} l^{\prime} i}^{\alpha}}{d_{0 l^{\prime} i}^{\alpha}}\right)^{2}\right)+\left(1+\frac{1}{\Delta \tau_{c}} \sum_{l^{\prime} \in \Psi_{D}} \sum_{i=1}^{K} \frac{d_{l^{\prime} l^{\prime} i}^{\alpha}}{d_{0 l^{\prime} i}^{\alpha}}+\frac{K(1-\Delta)}{\Delta \tau_{c}}+\frac{\sigma^{2}}{\Delta \rho \tau_{c}}\right)\left(K+\sum_{l^{\prime} \in \Psi_{D}} \sum_{i=1}^{K} \frac{d_{l^{\prime} l^{\prime} i}^{\alpha}}{d_{0 l^{\prime} i}^{\alpha}}+\frac{\sigma^{2}}{\rho}\right)\right) \\
& \leq \operatorname{SINR}_{0 k}^{\mathrm{SP}-\mathrm{UB}}=M(1-\Delta) /\left(\frac{M(1-\Delta)}{\tau_{c}} \sum_{l^{\prime} \in \Psi_{D}} \sum_{i=1}^{K}\left(\frac{d_{l^{\prime} l^{\prime} i}^{\alpha}}{d_{0 l^{\prime} i}^{\alpha}}\right)^{2}+\frac{M}{\tau_{c}} \frac{(1-\Delta)^{2}}{\Delta}\left(K+\sum_{l^{\prime} \in \Psi_{D}} \sum_{i=1}^{K}\left(\frac{d_{l^{\prime} l^{\prime} i}^{\alpha}}{d_{0 l^{\prime} i}^{\alpha}}\right)^{2}\right)\right. \\
& \left.+\frac{(1-\Delta)^{2}}{\tau_{c}^{2} \Delta}\left(K+\sum_{l^{\prime} \in \Psi_{D}} \sum_{i=1}^{K}\left(\frac{d_{l^{\prime} l^{\prime} i}^{\alpha}}{d_{0 l^{\prime} i}^{\alpha}}\right)^{2}\right)+\left(1+\frac{1}{\Delta \tau_{c}} \sum_{l^{\prime} \in \Psi_{D}} \sum_{i=1}^{K} \frac{d_{l^{\prime} l^{\prime} i}^{\alpha}}{d_{0 l^{\prime} i}^{\alpha}}+\frac{K(1-\Delta)}{\Delta \tau_{c}}+\frac{\sigma^{2}}{\Delta \rho \tau_{c}}\right)\left((1-\Delta)\left(K+\sum_{l^{\prime} \in \Psi_{D}} \sum_{i=1}^{K} \frac{d_{l^{\prime} l^{\prime} i}^{\alpha}}{d_{0 l^{\prime} i}^{\alpha}}\right)+\frac{\sigma^{2}}{\rho}\right)\right)
\end{aligned}
$$

\section{APPENDIX D \\ RESULTS FROM STOCHASTIC GEOMETRY}

Lemma 5. ([8]) The distribution of the distance $d_{l^{\prime} l^{\prime} i}$ between a UEs and its serving $B S$ for $l^{\prime} \in \Phi_{D}$ and $i \in\{1, \ldots, K\}$, where $\Phi_{D}$ is a homogeneous PPP with density $D$, is $d_{l^{\prime} l^{\prime} i} \sim$ Rayleigh $\left(\frac{1}{\sqrt{2 \pi D}}\right)$. Then we have that $\mathbb{E}\left\{d_{00 k}^{\alpha}\right\}=\frac{\Gamma(\alpha / 2+1)}{(\pi D)^{\alpha / 2}}$ for $\alpha>-2$.

Lemma 6. ([8, Appendix B]) For $\kappa \in\{1,2\}$ and $d_{l l^{\prime} i} \in \Psi_{D}$ being the distance between $\mathrm{BS}_{l}$ and $\mathrm{UE}_{l^{\prime} i}$ where $\Psi_{D}$ describes $a$ the set of BSs distributed as a homogeneous PPP with density $D$, we have $\forall i, j \in\{1, \ldots, K\}$

$$
\begin{aligned}
\mathbb{E}\left\{\sum_{l^{\prime} \in \Psi_{D}}\left(\frac{d_{l^{\prime} l^{\prime} i}^{\alpha}}{d_{0 l^{\prime} i}^{\alpha}}\right)^{\kappa}\right\} & =\frac{2}{\kappa \alpha-2} \\
\mathbb{E}\left\{\sum_{l^{\prime} \in \Psi_{D}} \sum_{l \in \Psi_{D} \backslash\left\{l^{\prime}\right\}} \frac{d_{l^{\prime} l^{\prime} i}^{\alpha}}{d_{0 l^{\prime} i}^{\alpha}} \frac{d_{l l j}^{\alpha}}{d_{0 l j}^{\alpha}}\right\} & =\left(\frac{2}{\alpha-2}\right)^{2} \\
\mathbb{E}\left\{\sum_{l^{\prime} \in \Psi_{D}} \frac{d_{l^{\prime} l^{\prime} i}^{\alpha}}{d_{0 l^{\prime} i}^{\alpha}} \frac{d_{l^{\prime} l^{\prime} j}^{\alpha}}{d_{0 l^{\prime} j}^{\alpha}}\right\} & \leq\left(\frac{1}{\alpha-1}\right) \text { for } i \neq j .
\end{aligned}
$$

\section{REFERENCES}

[1] D. Verenzuela, E. Björnson, and L. Sanguinetti, "Spectral efficiency of superimposed pilots in uplink massive MIMO systems," in Proc. IEEE GLOBECOM, Dec 2017, pp. 1-6.

[2] Cisco, "Visual networking index: Global mobile data traffic forecast update, 2014-2019," Tech. Rep., Feb. 2015.

[3] P. Popovski et al., "Mobile and wireless communications enablers for the twenty-twenty information society (METIS): Scenarios, requirements and KPIs for 5G mobile and wireless system," ICT317669-METIS/D1.1, Tech. Rep., May 2013, [Online] Available: http: //publications.lib.chalmers.se/records/fulltext/213055/local_213055.pdf.

[4] J. M. A. Fehske, G. Fettweis and G.Biczok, "The global footprint of mobile communications: The ecological and economic perspective," IEEE Trans. Commun., vol. 49, no. 8, pp. 55-62, 2011.

[5] R. Baldemair, E. Dahlman, G. Fodor, G. Mildh, S. Parkvall, Y. Selen, $\mathrm{H}$. Tullberg, and K. Balachandran, "Evolving wireless communications: Addressing the challenges and expectations of the future," IEEE Veh. Technol. Mag., vol. 8, no. 1, pp. 24-30, March 2013.

[6] H. Q. Ngo, E. G. Larsson, and T. L. Marzetta, "Energy and spectral efficiency of very large multiuser MIMO systems," IEEE Trans. Commun., vol. 61, no. 4, pp. 1436-1449, Apr. 2013.
[7] E. Björnson, L. Sanguinetti, J. Hoydis, and M. Debbah, "Optimal design of energy-efficient multi-user MIMO systems: Is massive MIMO the answer?" IEEE Trans. Wireless Commun., vol. 14, no. 6, pp. 3059-3075, Jun. 2015.

[8] E. Björnson, L. Sanguinetti, and M. Kountouris, "Deploying dense networks for maximal energy efficiency: Small cells meet massive MIMO," IEEE J. Sel. Areas Commun., vol. 34, no. 4, pp. 832-847, Apr. 2016.

[9] H. Yang and T. L. Marzetta, "Total energy efficiency of cellular large scale antenna system multiple access mobile networks," in Proc. IEEE OnlineGreenComm, Oct 2013, pp. 27-32.

[10] T. L. Marzetta, "Noncooperative cellular wireless with unlimited numbers of base station antennas," IEEE Trans. Wireless Commun., vol. 9 , no. 11 , pp. 3590-3600, 2010.

[11] T. L. Marzetta, E. G. Larsson, H. Yang, and H. Q. Ngo, Fundamentals of Massive MIMO. Cambridge Press, 2016.

[12] E. Björnson, J. Hoydis, and L. Sanguinetti, "Massive MIMO networks: Spectral, energy, and hardware efficiency," Foundations and Trends $\AA$ in Signal Processing, 2017. [Online]. Available: http://dx.doi.org/10.1561/2000000093

[13] H. Huh, G. Caire, H. C. Papadopoulos, and S. A. Ramprashad, "Achieving "massive MIMO" spectral efficiency with a not-so-large number of antennas," IEEE Trans. Wireless Commun., vol. 11, no. 9, pp. 32263239, September 2012.

[14] H. Yin, D. Gesbert, M. Filippou, and Y. Liu, "A coordinated approach to channel estimation in large-scale multiple-antenna systems," IEEE $J$. Sel. Areas Commun., vol. 31, no. 2, pp. 264-273, February 2013.

[15] E. Björnson, J. Hoydis, and L. Sanguinetti, "Massive MIMO has unlimited capacity," IEEE Trans. Wireless Commun., vol. 17, no. 1, pp. 574-590, Jan. 2018.

[16] J. Vinogradova, E. Björnson, and E. G. Larsson, "On the separability of signal and interference-plus-noise subspaces in blind pilot decontamination," in Proc. IEEE ICASSP, March 2016, pp. 3421-3425.

[17] H. Q. Ngo and E. G. Larsson, "EVD-based channel estimation in multicell multiuser MIMO systems with very large antenna arrays," in Proc. IEEE ICASSP, March 2012, pp. 3249-3252.

[18] R. R. Müller, L. Cottatellucci, and M. Vehkaperä, "Blind pilot decontamination," IEEE J. Sel. Topics Signal Process., vol. 8, no. 5, pp. 773-786, Oct 2014.

[19] Y. Li, Y.-H. Nam, B. L. Ng, and J. Zhang, "A non-asymptotic throughput for massive MIMO cellular uplink with pilot reuse," in Proc. IEEE GLOBECOM, Dec 2012, pp. 4500-4504.

[20] E. Björnson, E. Larsson, and M. Debbah, "Massive MIMO for maximal spectral efficiency: How many users and pilots should be allocated?" IEEE Trans. Wireless Commun., vol. 15, no. 2, pp. 1293-1308, 2016.

[21] R. Mochaourab, E. Björnson, and M. Bengtsson, "Adaptive pilot clustering in heterogeneous massive MIMO networks," IEEE Trans. Wireless Commun., vol. 15, no. 8, pp. 5555-5568, Aug 2016.

[22] P. Hoeher and F. Tufvesson, "Channel estimation with superimposed pilot sequence," in IEEE Proc. GLOBECOM, 1999, pp. 2162-2166.

[23] G. T. Zhou, M. Viberg, and T. McKelvey, "A first-order statistical method for channel estimation," IEEE Signal Process. Lett., vol. 10, no. 3, pp. 57-60, March 2003. 
[24] M. Dong, L. Tong, and B. M. Sadler, "Optimal insertion of pilot symbols for transmissions over time-varying flat fading channels," IEEE Trans. Signal Process., vol. 52, no. 5, pp. 1403-1418, May 2004.

[25] A. T. Asyhari and S. ten Brink, "Orthogonal or superimposed pilots? a rate-efficient channel estimation strategy for stationary MIMO fading channels," IEEE Trans. Wireless Commun., vol. 16, no. 5, pp. 27762789, May 2017.

[26] K. Upadhya, S. A. Vorobyov, and M. Vehkaperä, "Superimposed pilots are superior for mitigating pilot contamination in massive MIMO," IEEE Trans. Signal Process., vol. 65, no. 11, pp. 2917-2932, June 2017.

[27] K. Upadhya, S. A. Vorobyov, and M. Vehkaperä, "Downlink performance of superimposed pilots in massive MIMO systems," Submitted to IEEE Trans. Wireless Commun., 2016. [Online]. Available: http://arxiv.org/abs/1606.04476

[28] H. Zhang, S. Gao, D. Li, H. Chen, and L. Yang, "On superimposed pilot for channel estimation in multicell multiuser MIMO uplink: Large system analysis," IEEE Trans. Veh. Technol., vol. 65, no. 3, pp. 1492 1505, March 2016.

[29] X. Gao, O. Edfors, F. Rusek, and F. Tufvesson, "Massive MIMO performance evaluation based on measured propagation data," IEEE Trans. Wireless Commun., vol. 14, no. 7, pp. 3899-3911, July 2015.

[30] A. Pastore, T. Koch, and J. R. Fonollosa, "A rate-splitting approach to fading channels with imperfect channel-state information," IEEE Trans. Inf. Theory, vol. 60, no. 7, pp. 4266-4285, July 2014.

[31] S. M. Kay, Fundamentals of Statistical Signal Processing: Estimation Theory. Prentice Hall, 1993.

[32] R. M. Corless, G. H. Gonnet, D. E. G. Hare, D. J. Jeffrey, and D. E. Knuth, "On the LambertW function," Adv. in Comput. Math., vol. 5 , no. 1, pp. 329-359, 1996

[33] W. Lu and M. Di Renzo, "Stochastic geometry modeling of cellular networks: Analysis, simulation and experimental validation," in Proc. ACM MSWiM, 2015, pp. 179-188.

[34] S. Jacobsson, G. Durisi, M. Coldrey, U. Gustavsson, and C. Studer, "Throughput analysis of massive MIMO uplink with low-resolution ADCs," IEEE Trans. Wireless Commun., vol. 16, no. 6, pp. 4038-4051, Jun. 2017.

[35] C. Mollén, J. Choi, E. G. Larsson, and R. W. Heath, "Uplink performance of wideband massive MIMO with one-bit ADCs," IEEE Trans. Wireless Commun., vol. 16, no. 1, pp. 87-100, Jan 2017.

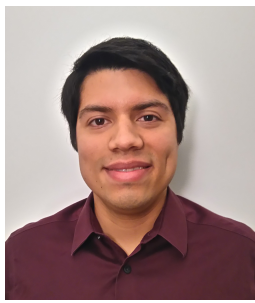

Daniel Verenzuela received the M.Sc. degree in Electrical Engineering with the double degree MERIT program from the Polytechnic of Turin (PdT), Turin, Italy and the Royal Institute of Technology (KTH), Stockholm, Sweden, in 2014, and the Licentiate of Engineering degree from Linköping University (LiU), Linköping, Sweden, in 2018. Currently, he is pursuing his Ph.D. degree at the Division of Communication Systems in the Department of Electrical Engineering (ISY) of Linköping University, Linköping, Sweden. His main research interest lies within the area of signal processing in multi-user Massive MIMO systems with emphasis in energy efficiency design.

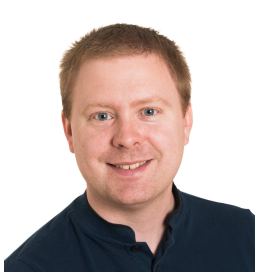

Emil Björnson (S'07-M'12-SM'17) received the M.S. degree in Engineering Mathematics from Lund University, Sweden, in 2007. He received the Ph.D. degree in Telecommunications from KTH Royal Institute of Technology, Sweden, in 2011. From 2012 to mid 2014, he was a joint postdoc at the AlcatelLucent Chair on Flexible Radio, SUPELEC, France, and at KTH. He joined Linköping University, Sweden, in 2014 and is currently Associate Professor and Docent at the Division of Communication Systems.

He performs research on multi-antenna communications, Massive MIMO, radio resource allocation, energy-efficient communications, and network design. He is on the editorial board of the IEEE Transactions on Communications (since 2017) and the IEEE Transactions on Green Communications and Networking (since 2016). He is the first author of the textbooks "Massive MIMO Networks: Spectral, Energy, and Hardware Efficiency" (2017) and "Optimal Resource Allocation in Coordinated MultiCell Systems" from 2013. He is dedicated to reproducible research and has made a large amount of simulation code publicly available.

Dr. Björnson has performed MIMO research for more than ten years and has filed more than ten related patent applications. He received the 2018 Marconi Prize Paper Award in Wireless Communications, the 2016 Best PhD Award from EURASIP, the 2015 Ingvar Carlsson Award, and the 2014 Outstanding Young Researcher Award from IEEE ComSoc EMEA. He also co-authored papers that received best paper awards at the conferences WCSP 2017, IEEE ICC 2015, IEEE WCNC 2014, IEEE SAM 2014, IEEE CAMSAP 2011, and WCSP 2009.

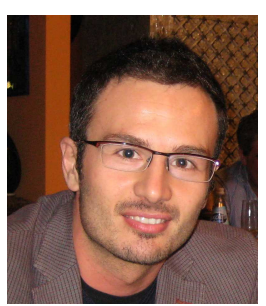

Luca Sanguinetti (SM'15) received the Laurea Telecommunications Engineer degree (cum laude) and the Ph.D. degree in information engineering from the University of Pisa, Italy, in 2002 and 2005 , respectively. Since 2005 he has been with the 'Dipartimento di Ingegneria dell'Informazione of the University of Pisa. In 2004, he was a visiting Ph.D. student at the German Aerospace Center (DLR), Oberpfaffenhofen, Germany. During the period June 2007 - June 2008, he was a postdoctoral associate in the Dept. Electrical Engineering at Princeton. During the period June 2010 - Sept. 2010, he was selected for a research assistantship at the Technische Universitat Munchen. From July 2013 to October 2017 he was with Large Systems and Networks Group (LANEAS), CentraleSupélec, Gif-sur-Yvette, France. He is currently an Associate Professor in the 'Dipartimento di Ingegneria dell'Informazione' of the University of Pisa, Italy.

Dr. Sanguinetti has co-authored the textbook Massive MIMO Networks: Spectral, Energy, and Hardware Efficiency (2017). He served as Exhibit Chair of the 2014 IEEE International Conference on Acoustics, Speech, and Signal Processing (ICASSP) and as the general co-chair of the 2016 Tyrrhenian Workshop on 5G\&Beyond. He served as an Associate Editor for IEEE TRANSACTIONS ON WIRELESS COMMUNICATIONS, and as Lead Guest Editor of IEEE JOURNAL ON SELECTED AREAS OF COMMUNiCATIONS Special Issue on "Game Theory for Networks" and as an Associate Editor for IEEE Journal on SELECTED AREAS of COMMUNICATIONS (series on Green Communications and Networking). Dr. Sanguinetti is currently serving as an Associate Editor for the IEEE SIgNAl PROCESSING LeTTERS and is a member of the Executive Editorial Committee of IEEE TRANSACTIONS ON WIRELESS COMMUNICATIONS.

His expertise and general interests span the areas of communications and signal processing. He received the 2018 Marconi Prize Paper Award in Wireless Communications and was the co-recipient of two best conference paper awards: IEEE WCNC 2013 and IEEE WCNC 2014. He was also the recipient of the FP7 Marie Curie IEF 2013 "Dense deployments for green cellular networks". 